THE STRUCTURAL SUITABILITY OF TENSEGRITY AIRCRAFT WINGS

\author{
Thesis \\ Submitted to \\ The School of Engineering of the \\ UNIVERSITY OF DAYTON
}

In Partial Fulfillment of the Requirements for

The Degree of

Master of Science in Mechanical Engineering

By

Austin S. Mills

Dayton, Ohio

May, 2020

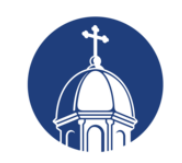

University of

Dayton 
Name: Mills, Austin S.

APPROVED BY:

David H. Myszka, Ph.D., P.E.

Advisory Committee Chairman

Associate Professor, Mechanical and

Aerospace Engineering
Andrew P. Murray, Ph.D.

Committee Member

Professor, Mechanical and Aerospace

Engineering

James J. Joo, Ph.D.

Committee Member

Advanced Structural Concepts Team

Lead, AFRL/RQVS

Robert J. Wilkens, Ph.D., P.E.

Eddy M. Rojas, Ph.D., M.A., P.E.

Associate Dean for Research and Innovation

Dean, School of Engineering

Professor

School of Engineering 


\section{ABSTRACT \\ THE STRUCTURAL SUITABILITY OF TENSEGRITY AIRCRAFT WINGS}

Name: Mills, Austin S.

University of Dayton

Advisor: Dr. David H. Myszka

This thesis presents an investigation of the suitability of tensegrity aircraft wing concepts and compares their simulated structural performance to a baseline conventional wing structure. Tensegrity systems, which consist of arrangements of struts and cables, are appealing for their structural efficiency, enabling lightweight structures with each member loaded in tension or compression. Of specific interest, tensegrity systems may provide a pathway to morphing aircraft structures through the actuation of cables. The present study compares two tensegrity-based wing designs to the aluminum Van's RV-4 aircraft rib/spar wing structure, chosen as the baseline performance case. Aerodynamic loading conditions are derived which simulate a $2 \mathrm{~g}$ pullup maneuver and a $1 \mathrm{~g}$ pushover, intended to interrogate the structures under characteristic positive and negative loading. The first tensegrity concept, developed with design judgment, is configured by merging known unit cells and is shown to yield deflections and strain energies comparable to the conventional wing at a fraction of its weight. The second tensegrity design, in contrast, is developed by application of a topology optimization algorithm, intended to minimize the weight with maximum stress and strain energy constraints. The topology-optimized wing has similar structural performance at slightly less weight than the designer-developed tensegrity wing. Additionally, a scaled down physical prototype of the designer-developed tensegrity wing was designed and fabricated, providing valuable insight into practical hurdles of tensegrity construction. 


\section{ACKNOWLEDGMENTS}

I'd like to start by thanking my advisor David Myszka for providing me the opportunity to work on this project. It's been a great experience and having the benefit of participating in research that challenges me and has introduced me to new fields is invaluable. Dave's dedication to excellence and vision for the project deeply inspired my passion for this thesis. He has been an outstanding mentor and has elevated my graduate study experience to a level I'll always remember and cherish.

The DIMLab and my fellow lab members have been nothing but supportive of my research efforts and I appreciate the companionships I've made along the way. After having a class with Drew Murray during my undergraduate I always saw him as one of the best professors Dayton had to offer. To have had the opportunity to go on and perform research alongside him during my graduate studies has been nothing short of a honor.

My gratitude also goes out to those of the Air Force Research Laboratory. James Joo, Daniel Woods, Zachary Montgomery, and many more were all very welcoming and supportive during my time at AFRL. A special thanks to Jared Neely for especially going out of his way for showing me around and helping with fabricating my tensegrity prototype.

Of course, a special tribute to those closest to me, my family and friends. Their endearing support and love keeps me going and I have had them to lean on whenever I have my doubts on my project. I'm grateful for everyone who has helped me achieve my goals and I'll appreciate these times forever. 


\section{TABLE OF CONTENTS}

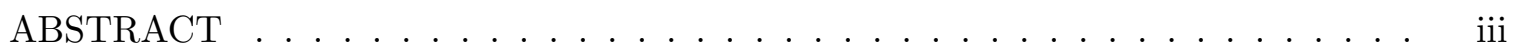

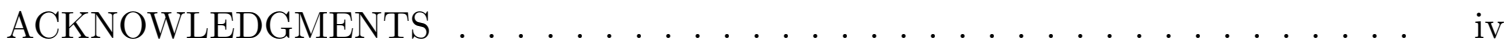

LIST OF FIGURES . . . . . . . . . . . . . . . . . . . . v vii

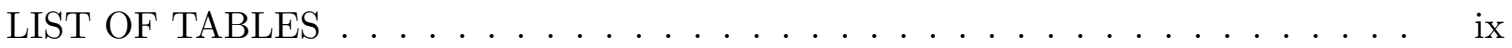

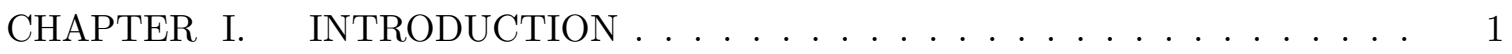

1.1 Tensegrity . . . . . . . . . . . . . . . . . . . 1

1.2 Aircraft Structures . . . . . . . . . . . . . . . . . . . 1

1.3 Motivation and Contribution . . . . . . . . . . . . . 4

1.4 Nomenclature. . . . . . . . . . . . . . . . . . . . 6

CHAPTER II. TENSEGRITY SYSTEMS FORMULATION . . . . . . . . . . 9

2.1 Geometry . . . . . . . . . . . . . . . . . . . . 11

2.2 Equilibrium and Pre-Tension . . . . . . . . . . . . . . . 12

2.3 Form-Finding . . . . . . . . . . . . . . . . . . . . . . 14

2.4 Stiffness . . . . . . . . . . . . . . . . . . . . . 15

2.5 Deflection, Strains and Stresses . . . . . . . . . . . . . . 15

2.6 Stability . . . . . . . . . . . . . . . . . 16

CHAPTER III. TOPOLOGY OPTIMIZATION . . . . . . . . . . . 18

3.1 Integers to Represent Candidate Member State . . . . . . . . . . . . . 19

3.2 Objective . . . . . . . . . . . . . . . . 20

3.3 Constraints . . . . . . . . . . . . . . . . 20

CHAPTER IV. METHODS OF ANALYSIS FOR WING COMPARISON STUDY 25

4.1 Skin Elements . . . . . . . . . . . . . . . . . . . . 25

4.2 Aerodynamic Loading Conditions . . . . . . . . . . . . . . . . 26

4.3 Load Interpolation to Structural Nodes . . . . . . . . . . . . . . . . 27

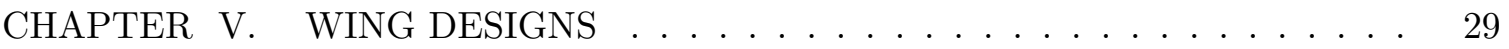

5.1 Baseline Conventional Wing . . . . . . . . . . . . . . . . . 29

5.2 Appropriate Nodal Locations for Tensegrity Wing Designs . . . . . . . . 30

5.3 Tensegrity Wing Developed through Designer Insights . . . . . . . . . . . 32

5.4 Tensegrity Wing Developed through Topology Optimization . . . . . . . 35

CHAPTER VI. TENSEGRITY WING PROTOTYPE DESIGN AND FABRICA-

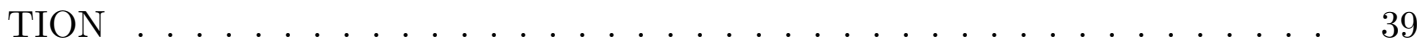

6.1 Class 6 Joint . . . . . . . . . . . . . . . . . . . . . . . . . 39

6.2 Class 1 Joint and Load Testing . . . . . . . . . . . . . . . . . 43

6.3 Full Assembly . . . . . . . . . . . . . . . . . . . . . 46 
6.4 Final Tensegrity Wing Fabrication . . . . . . . . . . . . . . . . . 49

CHAPTER VII. RESULTS AND DISCUSSION . . . . . . . . . . . . . . . 53

7.1 Analytical Wing Comparison . . . . . . . . . . . . . . . 53

7.2 Observations from the Tensegrity Wing Prototype Fabrication . . . . . . 55

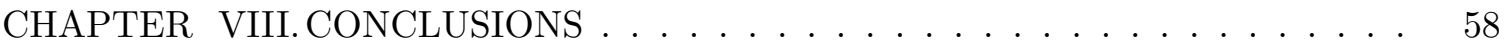

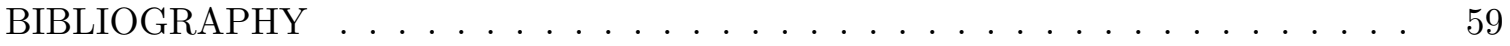




\section{LIST OF FIGURES}

1.1 Tensegrity Structures . . . . . . . . . . . . . . . . . 2

1.2 A conventional wing construction on a Vans RV aircraft (adapted from [14]). .

2.1 A spatial tensegrity Q-cross unit cell consisting of 7 nodes and 18 members (6 struts and 12 cables $). \ldots \ldots \ldots \ldots \ldots \ldots$

2.2 A system created from multiple instances of the Q-cross unit cell. . . . . . . . 10

2.3 A tensegrity system created from Q-cross unit cells that could serve as the structure for an aircraft wing. . . . . . . . . . . . . . . . 10

3.1 Example of $2 \mathrm{D}$ tensegrity topology optimization _ . . . . . . . . . . . 19

4.1 Abaqus STRI3 element with integration points . . . . . . . . . . . . 26

4.2 A diagram showing an aerodynamic force element $f_{y}$ in the $y$-direction, at an arbitrary location on the wing surface, and the four closest internal structural nodes, labeled $1,2,3$, and 4 . The dimensions $\beta_{z}$ and $\gamma_{z}$ are measured along the $z$-direction, and the dimensions $\beta_{x}$ and $\gamma_{x}$ are measured along the $x$-direction.

5.1 The conventional wing structure in Abaqus FEA, constructed to be representative of the notional RV-4 aircraft wing structure, for the models a with no skin and $\mathrm{b}$ with the skin. . . . . . . . . . . . . . . 30

5.2 Optimal location for 10 nodes to approximate a NACA 23015 airfoil. . . . . . . 31

5.3 Four R-cross unit cells are combined to form a tensegrity rib-like structure. Struts are shown in blue and cables are red. . . . . . . . . . . . . . . 32

5.4 Eight rib-like segments of Figure 5.3 are combined to form a tensegrity wing concept. . . . . . . . . . . . . . . . . . .

5.5 Ground structure mesh for the tensegrity topology optimization of a one-fifth span wing segment and corresponding optimization result. . . . . . . . . .

5.6 Topology-optimized tensegrity wing concept consisting of five combined wing segments of Figure $5.5 \ldots \ldots \ldots \ldots$. . . . . . . . . . . . . . . . . . .

6.1 Class 6 tensegrity joint base design . . . . . . . . . . . . . . . 40

6.2 Preliminary designs for joint cable connectivity . . . . . . . . . . . . . 41 
6.3 Class 6 joint design with safety wire guides . . . . . . . . . . . . 42

6.4 Finalized general joint design . . . . . . . . . . . . . . . . . . . . 43

6.5 Preliminary ideas for class 1 joints . . . . . . . . . . . . . . . . 44

6.6 Selected idea for class 1 joints . . . . . . . . . . . . . . . . . . 44

6.7 Design strength testing . . . . . . . . . . . . . . . . . 45

6.8 Finalized tensegrity wing section design. Colors indicate the condition of the cable connections as being in-line (blue), offset (orange), or sharing a hook (purple). 47

6.9 Finalized full tensegrity wing design . . . . . . . . . . . . . . . 48

6.10 Fabricated tensegrity wing section $\ldots \ldots \ldots \ldots$

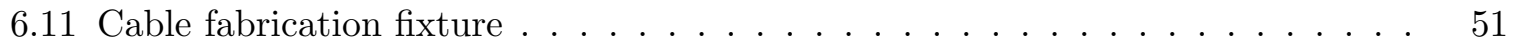

6.12 Updated cable fabrication fixture . . . . . . . . . . . . . . . 52

7.1 The displacements of the topology-optimized tensegrity wing under the $+2 \mathrm{~g}$ load

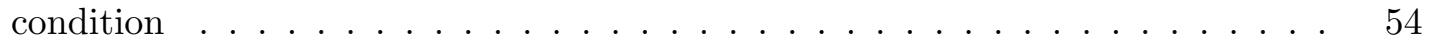

7.2 The vertical displacements of the conventional wing structure subjected to the $+2 \mathrm{~g}$ load condition, for the models (a) with no skin and (b) with the skin. . . . 


\section{LIST OF TABLES}

5.1 Cable diameters used in the designer-developed tensegrity aircraft wing. . . . .

5.2 Cable diameters used in the topology-optimized tensegrity aircraft wing. . . . .

7.1 Structural comparison between the conventional wing, designer-developed tensegrity wing, and topology optimization wing. . . . . . . . . . . . 


\section{CHAPTER I \\ INTRODUCTION}

\subsection{Tensegrity}

Tensegrity systems consist of a series of compressed struts connected by tensioned cables that place the system in a self-equilibrium state $[27,39]$. With all components being loaded axially (i.e., no bending or torsion), tensegrity structures can endure external loads with high stiffness and low stresses. Originally dating back to the 1940s, though the term was coined in the 1960s, tensegrity was most famously used by Buckminster Fuller [10] and Kenneth Snelson [28] to create exotic artistic pieces and architectures. Figures 1.1b and 1.1c showcase some of Snelson's tensegrity work. Tensegrity has since gone on to be utilized in numerous engineering applications [26]. Large tensegrity structures such as bridges and roofs benefit from the high strength to weight ratio tensegrity provides. Kurilpa Bridge shown in Figure 1.1a serves as the world's largest hybrid tensegrity bridge, producing a lightweight but strong structure while still being considered a stunning architecture. Additionally, research into areas such as vibration damping and bio-inspired designs continue to spur innovation $[1,17]$

\subsection{Aircraft Structures}

Aircraft wings must, at a basic level, provide the lift needed for flight while supporting the aircraft's weight and inertial loading throughout the flight path. As shown in the example in Figure 1.2, conventional wing structures are made up of spars running spanwise and ribs running chordwise $[21,14]$. In this arrangement, the ribs support the airfoil shape

and transfer loading to the spars, and the spars serve as the main structural members 


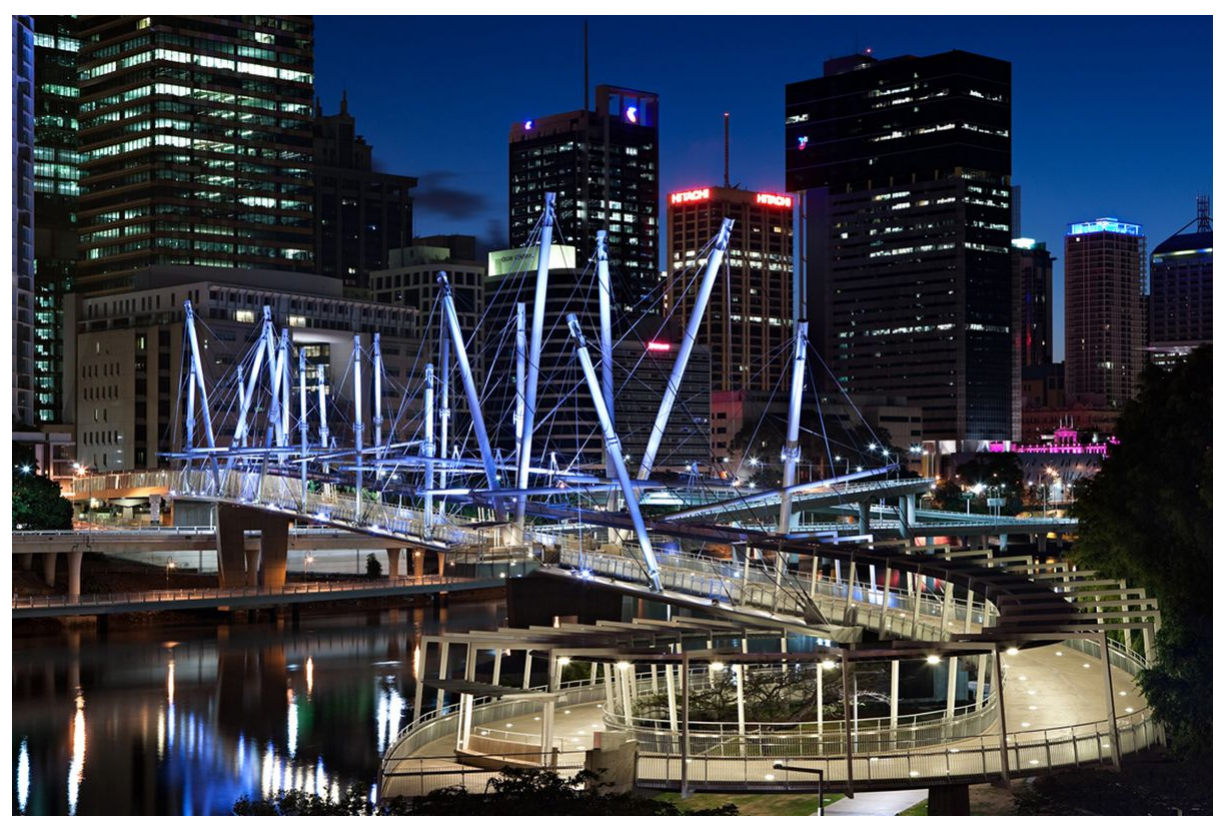

(a) Kurilpa Bridge in Brisbane, Queensland, Australia [25]

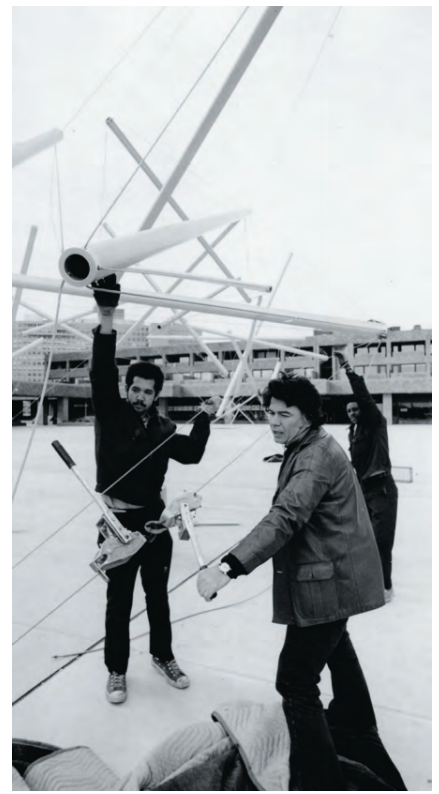

(b) Installation Ex. [12]

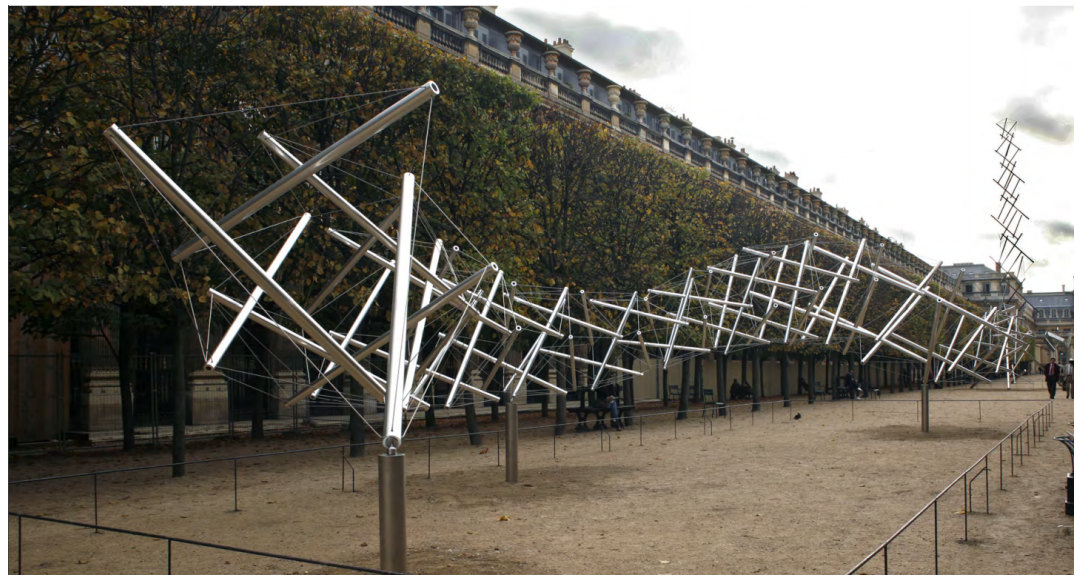

(c) Snelson's Sleeping Dragon, 2003 [12]

Figure 1.1: Tensegrity Structures 
extending from the fuselage. The skin then covers the exterior to transfer flight loads to the
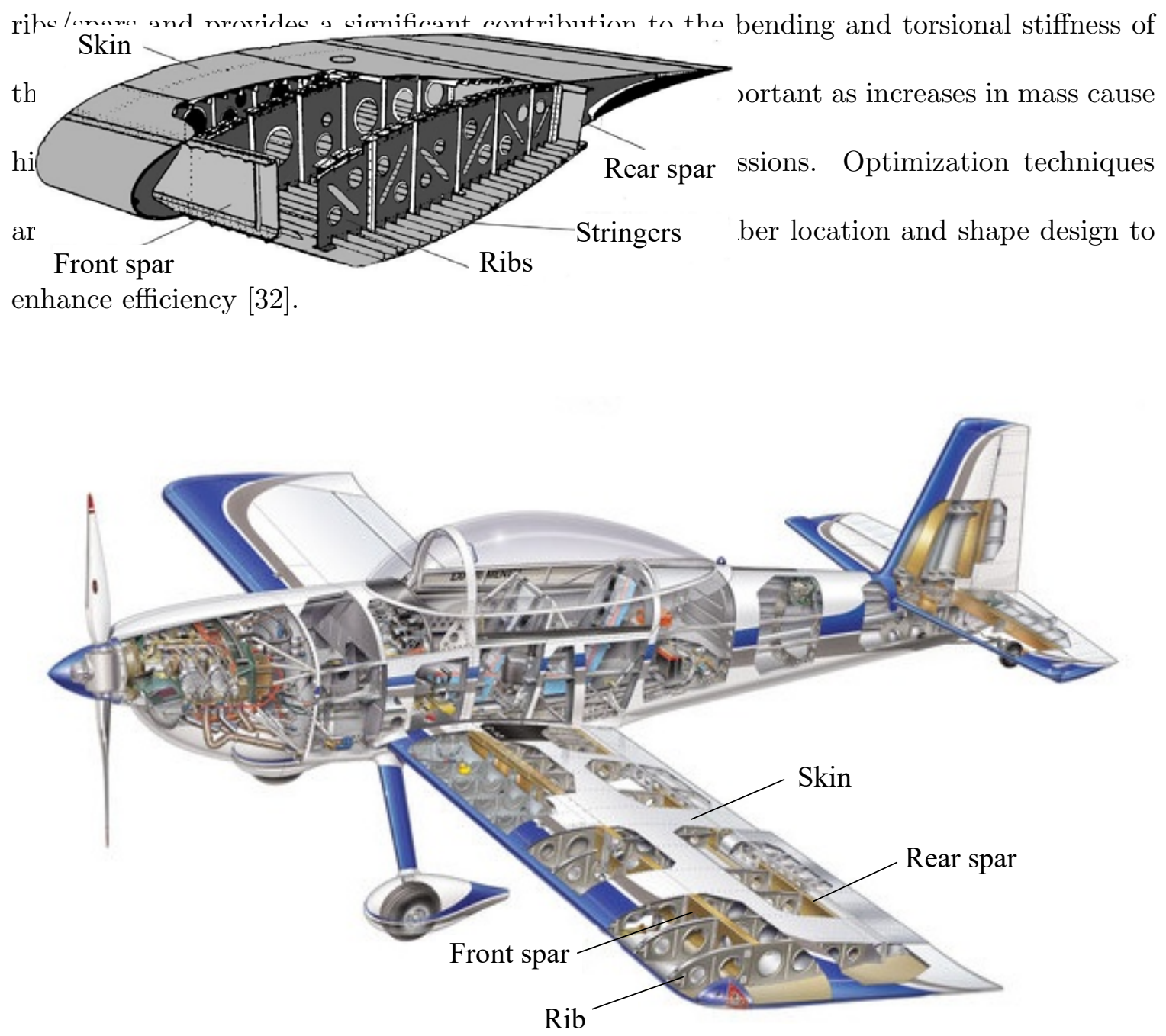

Figure 1.2: A conventional wing construction on a Vans RV aircraft (adapted from [14]).

Essentially all modern aircraft structural design processes use finite element analysis, and many also implement optimization algorithms [6]. A large array of loading conditions must be considered in the design of a wing structure, including high-g maneuvers, gusts, landing conditions, control surface inputs, vibrational environments and couplings (e.g., aeroelastic flutter margins), and engine thrust/drag. High-g maneuvers often, along 
with gust conditions, exert the greatest loads an aircraft experiences. Pullup maneuvers, for example, increase the aircraft pitch rate and generate an upward loading on the wing. Conversely, a pushover maneuver is associated with a decrease in aircraft pitch rate and generates downward wing loading. In the design process for a wing structure, an optimization algorithm may use the critical load cases to size or propagate discretized elements to fill the wing structure design space. With optimization objectives often posed for efficient use of material by either reducing weight, minimizing structural compliance, or providing beneficial aerodynamic wing shapes $[29,32]$, the optimizer seeks to utilize certain elements and remove others based on those desired objectives.

\subsection{Motivation and Contribution}

Morphing mechanisms allow mechanical systems to alter their geometric shape in a controlled manner. There is a long history of interest in mechanisms that change the shape of an aircraft wing. Schemes that change the shape of in-flight vehicle features seek to resolve conflicts that arise in different air vehicle speed regimes. Principal design criteria for aircraft structures are low weight, high strength and high rigidity. In contrast, morphing mechanisms are inherently compliant in order to permit motion. Accordingly, a fundamental challenge in creating morphing aircraft systems is developing an underlying structure that maintains strength and rigidity but permits motion. Tensegrity systems have been proposed as a concept that addresses this technical challenge.

As stated, tensegrity structures exhibit high stiffness-to-weight and strength-to-weight ratios. The mechanics of tensegrity systems have been thoroughly explored, including equilibrium [34], stiffness [11], pre-load [23], and stability [40]. In addition, Myszka and Joo completed a study which demonstrates that tensegrity systems have similar structural effi- 
ciency as trusses and I-beams [19]. Unlike a truss or I-beam, however, a tensegrity system has the capability to alter its shape by changing strut or cable length $[4,16]$, which may enable elegant pathways to aircraft morphing.

Tensegrity design involves determining an efficient strut and cable topology (i.e., connectivity) to exist within a given geometry. Large tensegrity systems can be configured from basic unit cells by using designer intuition. Alternatively, Ehara and Kanno [7] introduced a topology optimization based on the ground structure method and formulated the objective and constraints as a mixed integer linear programming (MILP) problem for efficient solution. Kanno [15] expanded the method to include stiffness and stress requirements. Xu et al.[37] expanded the method by including alternative objective functions and practiceoriented requirements, while Myszka et al. [20] included constraints that enable morphing. Tensegrity wing design through both approaches, built up from basic unit cells and through topology optimization, are explored in the present work.

The main contribution of this work is a structural comparison of two tensegrity wing structures to a conventional baseline wing. One tensegrity wing is designed by using designer intuition and standard design practices, and the second is the result of a topology optimization. Additionally a physical scaled-down prototype of a tensegrity wing was fabricated as a proof of concept. The remainder of the thesis is organized as follows. Tensegrity system mechanics approaches are reviewed in Section II. Section III presents the topology optimization employed. Section IV describes the analysis methodology used for the current comparison study. The three wing designs and their formulations are then detailed in Section V. Section VI shows the design and fabrication of the proof of concept tensegrity wing prototype. Section VII provides a discussion on the structural performance of each wing as well as the outcome of the prototype. Conclusions are drawn in Sect. VIII. 


\subsection{Nomenclature}

\section{Symbols}

$\alpha^{p} \quad$ set of coplanar members in the $p$ th plane for topology optimization

$\lambda$ topology optimization sufficiently large constant

$\mathbb{C}$ set of candidate cables for topology optimization

$\mathbb{E}$ set of eliminated members for topology optimization

$\mathbb{J} \quad$ set of indices of nodal degrees of freedom for topology optimization

$\mathbb{M} \quad$ set of candidate members for topology optimization

$\mathbb{M}^{c} \quad$ set of pairs of intersecting members for topology optimization

$\mathbb{N} \quad$ set of candidate nodes for topology optimization

$\mathbb{S} \quad$ set of candidate struts for topology optimization

A equilibrium matrix b topology optimization vector of taut cables

C connectivity matrix

c topology optimization vector of cables

d vector of nodal displacements

E node force density matrix

e vector of member elongations

F $\quad$ system force matrix

f vector of externally applied forces

K $\quad$ stiffness matrix

L member length diagonal matrix

$1 \quad$ vector of member lengths

M system member matrix

m vector of member connectivity

$\mathbf{N} \quad$ system node matrix

$\mathbf{n}$ vector of nodal coordinates

Q member force density diagonal matrix 
s topology optimization vector of $\bar{k}$ topology optimization member struts elastic stiffness

t vector of member forces

$\rho \quad$ material density

U $\quad$-direction member coordinate dif-

$\sigma^{u b} \quad$ topology optimization stress limit

ference diagonal matrix

a member cross-sectional area

$\mathbf{u} \quad$ vector of $\mathrm{x}$-direction member coor-

$b \quad$ topology optimization tautness

dinate difference

variable

V y-direction member coordinate dif-

ference diagonal matrix

c topology optimization cable vari-

able

$\mathbf{v} \quad$ vector of $\mathrm{y}$-direction member coor-

dinate difference

d nodal displacement

$e \quad$ member elongation

W z-direction member coordinate dif-

ference diagonal matrix

$f \quad$ externally applied force

w vector of z-direction member coor-

$l \quad$ member length

dinate difference

$m \quad$ number of members

$\mathrm{x} \quad$ vector of $\mathrm{x}$-axis nodal coordinates

$n \quad$ number of nodes

$\mathbf{y} \quad$ vector of $\mathrm{y}$-axis nodal coordinates

$n^{m} \quad$ number of internal mechanisms

$\mathbf{z} \quad$ vector of $\mathrm{z}$-axis nodal coordinates

$n^{s} \quad$ number of independent states of

U strain energy

prestress

$\mathcal{U}^{u b}$ topology optimization strain en-

ergy limit

$P \quad$ topology optimization label set of coplanar members

$\overline{\mathbf{K}}$ member elastic stiffness diagonal

$q \quad$ member force density

matrix

$r^{\mathrm{A}} \quad$ rank of $\mathbf{A}$ 
$S$

topology optimization strut vari-

able

$t \quad$ member force

$x \quad$ X-axis nodal coordinate

$Y \quad$ elastic modulus

$y \quad \mathrm{y}$-axis nodal coordinate

z $\quad$ z-axis nodal coordinate

$\boldsymbol{\sigma} \quad$ vector of member stress

\section{Subscripts}

$0 \quad$ initial

c cable e elastic

$F \quad$ free

$g \quad$ geometric

$i \quad$ node index of summation

j node index of summation

$k \quad$ member index of summation

$k^{\prime} \quad$ member index of summation

$R \quad$ restrained

$r \quad$ node index of summation

$s \quad$ strut 


\section{CHAPTER II}

\section{TENSEGRITY SYSTEMS FORMULATION}
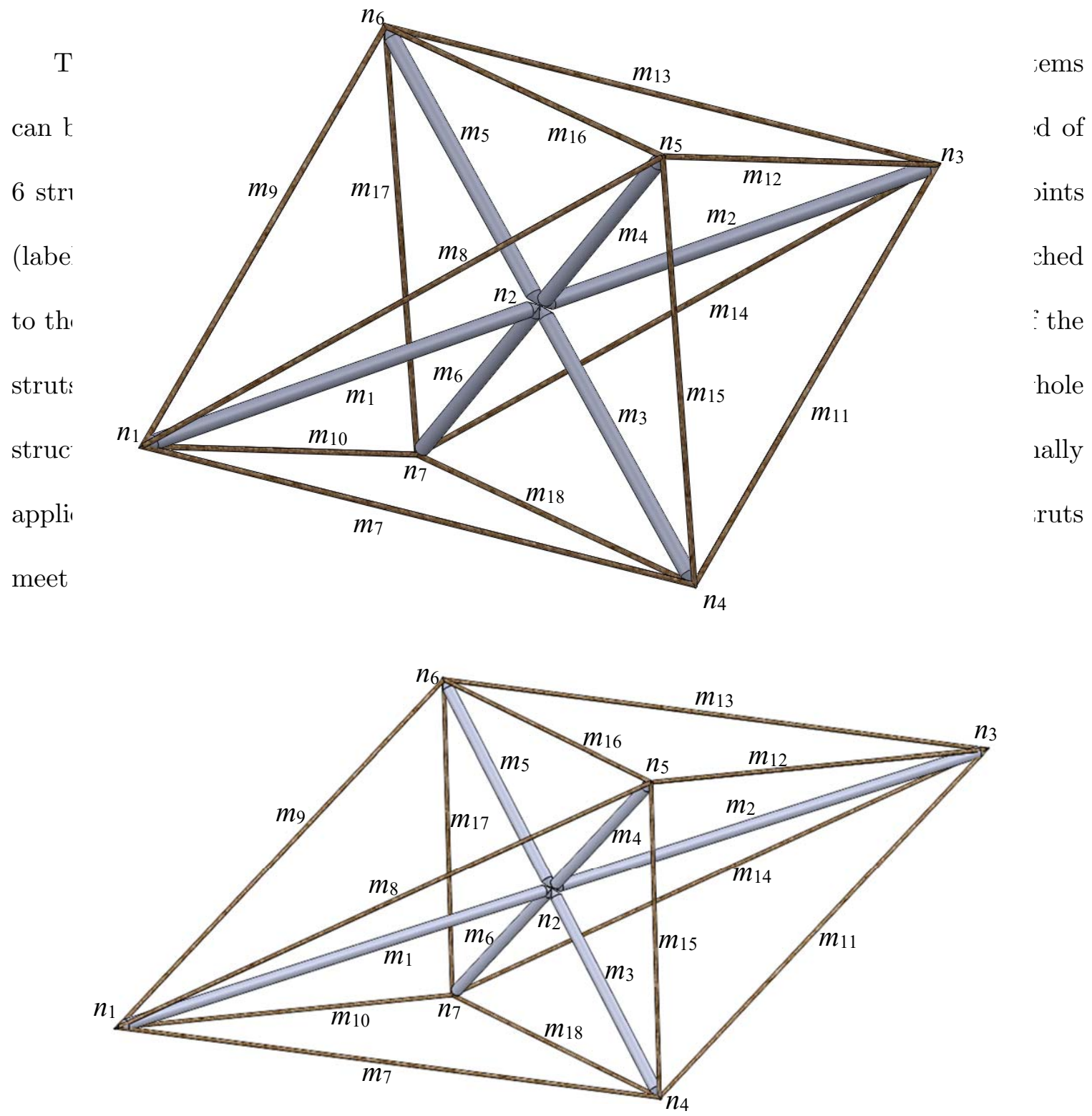

Figure 2.1: A spatial tensegrity Q-cross unit cell consisting of 7 nodes and 18 members (6 struts and 12 cables).

The tensegrity system in Figure 2.1 is termed a quadrilateral cross, or Q-cross, which can serve as a unit cell. The Q-cross is a minimal tensegrity, meaning that it has the fewest 
number of cables required to stabilize the unit cell. A larger tensegrity system is commonly comprised of multiple unit cells. Figure 2.2 shows four instances of Q-cross cells, sized and arranged in a manner that could represent a section of an aircraft wing, analogous to the wing section shown in Figure 1.2. Notice that additional, redundant cables have been added to join the unit cells into a cohesive tensegrity system. Figure 2.3 further duplicates the unit cells in an arrangement that could represent an entire aircraft wing.

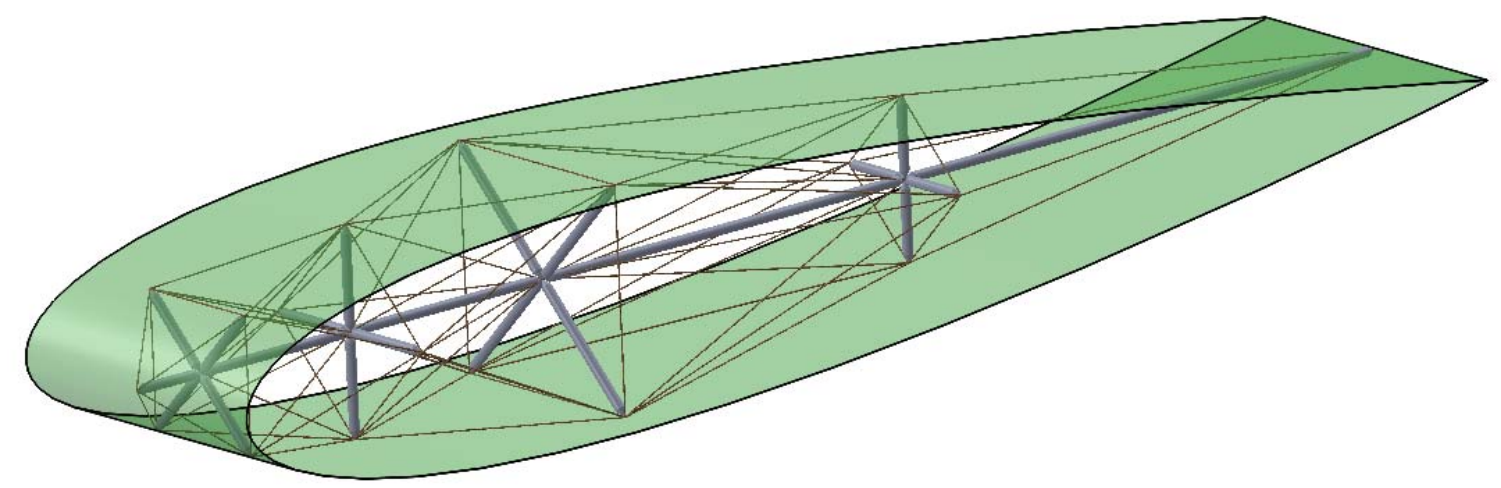

Figure 2.2: A system created from multiple instances of the Q-cross unit cell.

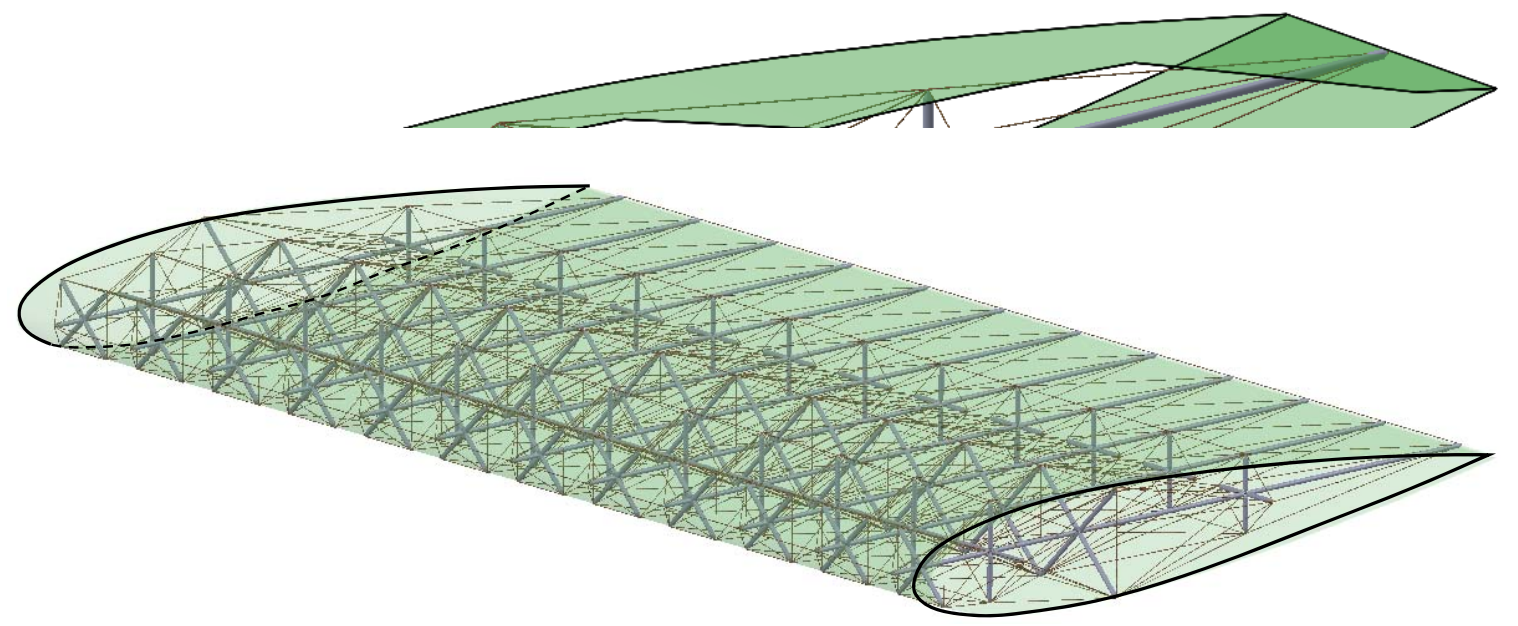

Figure 2.3: A tensegrity system created from Q-cross unit cells that could serve as the structure for an aircraft wing. 
A substantial amount of theory has been developed to describe the mechanics of tensegrity systems $[27,39]$. The fundamental concepts used in the analysis presented in Sec. 3 are reviewed below for completeness.

\subsection{Geometry}

A general tensegrity framework is composed of $n$ nodes joined by $m$ members. The coordinates of node $i$ are represented by the vector $\mathbf{n}_{i}=\left\{x_{i}, y_{i}, z_{i}\right\}^{T}$. The system node matrix is defined as $\mathbf{N}=\left[\begin{array}{llll}\mathbf{n}_{1} & \mathbf{n}_{2} & \ldots & \mathbf{n}_{n}\end{array}\right], \in \mathbb{R}^{3 \times \mathbf{n}}$. It is convenient to arrange the nodal coordinates as $\mathbf{x}=\left\{x_{1}, x_{2}, \ldots x_{n}\right\}^{T}, \in \mathbb{R}^{\mathrm{n} \times 1}$, and similarly with $\mathbf{y}$ and $\mathbf{z}$. Thus, the directional coordinate vectors and system node matrix are related by

$$
\mathbf{N}=\left(\begin{array}{c}
\mathbf{x}^{T} \\
\mathbf{y}^{T} \\
\mathbf{z}^{T}
\end{array}\right)
$$

For the $k^{\text {th }}$ member that connects node $i$ to node $j$, a member vector is $\mathbf{m}_{k}=\left\{\mathbf{n}_{i}-\mathbf{n}_{j}\right\}, \in$ $\mathbb{R}^{3 \times 1}$. A system member matrix is assembled as $\mathbf{M}=\left[\begin{array}{llll}\mathbf{m}_{1} & \mathbf{m}_{2} & \ldots & \mathbf{m}_{m}\end{array}\right], \in \mathbb{R}^{3 \times m}$.

The connectivity of the structure specifies how the members connect the nodes. Again considering member $k$, which connects node $i$ to node $j$, the $(k, r)$ element in the connectivity matrix is

$$
C_{(k, r)}=\left\{\begin{array}{cl}
1, & \text { if } r=i \\
-1, & \text { if } r=j \\
0, & \text { otherwise }
\end{array}, \quad r=1, \ldots, n .\right.
$$

Replicating Eq. (2.2) for each member $(k=1, \ldots, m)$ results in a full connectivity matrix $\mathbf{C} \in \mathbb{R}^{\mathrm{m} \times \mathrm{n}}$.

Coordinate difference vectors $\mathbf{u}, \mathbf{v}$, and $\mathbf{w}$ in the $x-, y$-, and $z$-directions, respectively, provide the directional components of each member,

$$
\mathbf{u}=\mathbf{C} \mathbf{x}, \quad \mathbf{v}=\mathbf{C} \mathbf{y}, \quad \mathbf{w}=\mathbf{C} \mathbf{z}, \in \mathbb{R}^{\mathrm{m} \times 1}
$$


The coordinate difference vectors and member matrix are related by

$$
\mathbf{M}=\left(\begin{array}{c}
\mathbf{u}^{T} \\
\mathbf{v}^{T} \\
\mathbf{w}^{T}
\end{array}\right)
$$

A member length vector $\mathbf{l} \in \mathbb{R}^{\mathrm{m} \times 1}$ is generated, such that for the $k^{\text {th }}$ member, $l_{k}=$ $\left\|\mathbf{m}_{k}\right\|=\sqrt{u_{k}^{2}+v_{k}^{2}+w_{k}^{2}}$. For computation efficiency, the coordinate difference and member length vectors are converted to diagonal matrices,

$$
\begin{aligned}
\mathbf{U}=\operatorname{diag}(\mathbf{u}), \quad \mathbf{V}=\operatorname{diag}(\mathbf{v}), \\
\mathbf{W}=\operatorname{diag}(\mathbf{w}), \quad \mathbf{L}=\operatorname{diag}(\mathbf{l}) .
\end{aligned}
$$

\subsection{Equilibrium and Pre-Tension}

The magnitude of the internal force in member $k$ is designated as $t_{k}$. Throughout the use of a tensegrity system, care must be taken to ensure that cables remain in tension $(t>0)$ and the struts remain in compression $(t<0)$. The vector of member forces is $\mathbf{t} \in \mathbb{R}^{\mathrm{m} \times 1}$, with $\mathbf{t}_{0}$ being the initial forces (pre-tension).

External forces applied to node $i$ are represented by the vector $\mathbf{f}_{i}=\left\{f_{i_{x}}, f_{i_{y}}, f_{i_{z}}\right\}^{T}$. A system force matrix is defined as $\mathbf{F}=\left[\begin{array}{llll}\mathbf{f}_{1} & \mathbf{f}_{2} & \ldots & \mathbf{f}_{n}\end{array}\right], \in \mathbb{R}^{3 \times n}$, and a system force vector is $\mathbf{f}=\operatorname{vec}(\mathbf{F}) \in \mathbb{R}^{3 \mathrm{n} \times 1}$.

Equating forces at each node [39], equilibrium equations are assembled as

$$
\mathbf{A} \mathbf{t}=\mathbf{f}
$$

where the equilibrium matrix is

$$
\mathbf{A}=\left(\begin{array}{c}
\mathbf{C}^{T} \mathbf{U L}^{-1} \\
\mathbf{C}^{T} \mathbf{V} \mathbf{L}^{-1} \\
\mathbf{C}^{T} \mathbf{W} \mathbf{L}^{-1}
\end{array}\right) \in \mathbb{R}^{3 \mathrm{n} \times \mathrm{m}}
$$

An initial tension is applied to the cables of a tensegrity system prior to any externally applied loads. This pre-tension in the cables results in compression on the struts to create 
the stable, self-equilibrium state. Consequently, the equilibrium condition for the tensegrity system prior to any application of external loads is

$$
\mathbf{A} \mathbf{t}_{0}=0
$$

Note that for self-equilibrium, the pre-tension on the cables (and pre-compression in the struts) are related such that $\mathbf{t}_{0}$ lies in the null space of $\mathbf{A}$. The Modified Maxwell's Rule identifies that a tensegrity system has $n^{s}$ degree of static indeterminacy or independent states of self-stress [24],

$$
n^{s}=m-r^{\mathrm{A}}
$$

where $r^{\mathrm{A}}$ is the rank of $\mathbf{A}$. If $n^{s}=0$, the system is statically determinate and pre-tension cannot be applied to the system.

Further, a $n^{m}$ number of internal independent mechanisms or degree of kinematic indeterminacy [39] is determined by

$$
n^{m}=3 n-6-r^{\mathrm{A}}
$$

for a 3-dimensional spatial structure. Likewise for the planar case, the number of internal independent mechanisms is

$$
n^{m}=2 n-3-r^{\mathrm{A}}
$$

A mechanism refers to the ability for nodal motion, classified by infinitesimally small motion or larger finite motion. Eq. (2.10) and Eq. (2.11) cannot be used to differentiate between infinitesimal and finite mechanisms. From the standpoint of a realistic structure every node should have multiaxial stiffness and as such the structure should be kinematically determinate, $n^{m}=0$.

To determine pre-tension relationships, it is convenient to define force density as the internal force per unit length of the member $k, q_{k}=t_{k} / l_{k}$. A force density matrix is 
constructed as $\mathbf{Q}=\operatorname{diag}\left(\left[q_{1}, q_{2}, \ldots, q_{m}\right]\right) \in \mathbb{R}^{\mathrm{m} \times \mathrm{m}}$. As with member forces, the initial pre-tensioned force density matrix is $\mathbf{Q}_{0}$.

Equivalent to Eq. (2.6), equilibrium conditions can be written [27] as

$$
\mathbf{M ~ Q ~ C ~}=\mathbf{F},
$$

and in the unloaded state

$$
\mathbf{M} \mathbf{Q}_{0} \mathbf{C}=0 .
$$

Thus, Eq. (2.13) will generate $3 n$ equations in terms of $q_{k}, k=1, \ldots, m$ that must be satisfied for the tensegrity system to be in self-equilibrium. Being a non-square (overdetermined) system of equations, a realizable tensegrity system will produce a consistent set of solutions that satisfy all the redundant equations.

\subsection{Form-Finding}

The analysis process outlined to this point presumes that the nodal locations are known. A tensegrity system will not generally be stable for arbitrary node locations. The selfequilibrium condition for each direction [39] is

$$
\mathbf{E x}=0, \quad \mathbf{E y}=0, \quad \mathbf{E z}=0,
$$

where a node force density matrix is

$$
\mathbf{E}=\mathbf{C}^{T} \mathbf{Q} \mathbf{C}, \quad \in \mathbb{R}^{\mathrm{n} \times \mathrm{n}}
$$

The nodal coordinates $\mathbf{x}, \mathbf{y}, \mathbf{z}$ cannot be uniquely determined by solving Eq. (2.14) as $\mathbf{E}$ of a tensegrity system is always rank deficient, and therefore, not invertible.

Determining the relationship between the strut and cable lengths and the nodal coordinates of the self-equilibrium state of a tensegrity system is known as form-finding. Tibert 
and Pelligrino [30] outline several alternative form-fitting methods. A physically identifiable method involves maintaining constant strut lengths and varying the cable lengths until a minimum is achieved. This will be the lowest energy, and hence stable, configuration. The study outlined in this thesis investigates unit cells with a high level of symmetry. Consequently, obtaining nodal coordinates that minimize cable lengths can be done analytically.

\subsection{Stiffness}

The stiffness matrix of a tensegrity system is computed as,

$$
\mathbf{K}=\mathbf{K}_{e}+\mathbf{K}_{g}, \quad \in \mathbb{R}^{\mathrm{m} \times \mathrm{m}}
$$

where

$$
\mathbf{K}_{e}=\mathbf{A} \overline{\mathbf{K}} \mathbf{A}^{T}, \quad \mathbf{K}_{g}=\mathbf{I}_{3} \otimes \mathbf{E},
$$

with $\overline{\mathbf{K}}=\operatorname{diag}\left(\left[Y_{1} a_{1} / l_{1}, \ldots, Y_{m} a_{m} / l_{m}\right]\right), Y_{k}$ is the modulus of elasticity of member $k, a_{k}$ is the cross sectional area of member $k, \mathbf{I}_{3}$ is the $3 \times 3$ identity matrix, and $\otimes$ is the tensor (or Kronecker) product. The first stiffness component in Eq. (2.16), termed the elastic stiffness matrix $\mathbf{K}_{e}$, results from the member size and material properties. The second stiffness component, termed the geometrical stiffness matrix $\mathbf{K}_{g}$, is related to the pre-tension of the members.

\subsection{Deflection, Strains and Stresses}

Once the stiffness matrix is obtained, the deflection of the nodes due to externally applied forces can be determined. Consistent with the system force vector $\mathbf{f}$, the nodal deflection vector is defined as $\mathbf{d}=\left\{d_{1_{x}}, \ldots, d_{n_{x}}, d_{1_{y}}, \ldots, d_{n_{y}}, d_{1_{z}}, \ldots, d_{n_{z}},\right\}^{T}, \in \mathbb{R}^{3 \mathrm{n} \times 1}$. Then,

$$
\mathbf{K d}=\mathbf{f} .
$$


Note that the solution to Eq. (2.18) involves a nonlinear analysis as deflections alter the node locations, and subsequently the stiffness matrices. It is common in tensegrity systems to gradually apply the forces in small increments, ensuring that at each step the equilibrium of the tensegrity system in Eq. (2.14) is satisfied and the stiffness matrices in Eqs. (2.16) and (2.17) are appropriately updated.

Equation (2.6) is used to determine the load in each member. Similarly, a vector of member elongations $\mathbf{e}$ is

$$
\mathbf{e}=\mathbf{A}^{T} \mathbf{d}
$$

and a vector of the member stress $\boldsymbol{\sigma}$ is

$$
\boldsymbol{\sigma}=\overline{\mathbf{K}} \mathbf{e}
$$

In elastic structures carrying static loads, the external work imparted onto the system and strain energy are equal. Thus, the strain energy stored within the tensegrity system due to external loads is

$$
\mathcal{U}=\frac{1}{2}\left(\mathbf{t}-\mathbf{t}^{\mathbf{0}}\right)^{T} \mathbf{e}=\frac{1}{2} \mathbf{f}^{T} \mathbf{d}
$$

\subsection{Stability}

Equilibrium does not guarantee stability. A tensegrity system is stable if its potential energy is at a local strict minimum. Thus, the tensegrity will return to its equilibrium position after being subjected to a small disturbance. After restraining rigid-body motions, a tensegrity is stable if

$$
\mathbf{d}^{T} \mathbf{K d}>0
$$

or more generally, if the stiffness matrix $\mathbf{K}$ is positive definite. A tensegrity structure with finite or infinitesimal mechanisms is typically not stable and as such fails the Eq. (2.22) check 
for stability. There are known instances in which tensegrity structures with mechanisms may react in a stable manner based on the direction of loading applied. For instance if a three-dimensional node within a tensegrity structure is rigid in-plane but has no out-ofplane stiffness it has an mechanism, and as long as the applied loading doesn't cause the node to displace out-of-plane then the structure will appear stable. 


\section{CHAPTER III}

\section{TOPOLOGY OPTIMIZATION}

Topology optimization allows the use of computation to determine an efficient use of material within a design space given a set of loads and boundary conditions. Conventional topology optimization uses the finite element method to determine optimal material distribution, often by solving for material densities of discrete elements representing the design space. Tensegrity topology optimization, similar to truss topology optimization, most often undergoes shape or sizing optimizations in addition to the initial topology optimization [2]. Shape optimization takes the given topology connectivity and moves nodal coordinates in an optimal fashion. Similarly, sizing optimization involves taking the given topology optimization and finding optimal cross-sectional areas for the members. The work outlined in this thesis uses the established topology optimization procedure for tensegrity systems with compliant and stress constraints, which was presented by Kanno [15]. Sizing optimization of the member cross-sectional areas is performed following the topology optimization. The optimization formulation is reviewed in this section.

Using a ground structure approach, the location of all nodes $\left(\mathbf{n}_{i}, i=1, \ldots, n\right)$ are specified as follows. The design space is the region within which the optimized tensegrity system is contained. Nodes must be placed at all prescribed loads and rigid-body motion restraints. Additional nodes are distributed to adequately fill the design space. All node pairs are connected with a candidate member. Unlike Truss-based ground structure methods in which overlapping members are permitted; overlapping candidate members are eliminated for tensegrity systems as the long members cannot always be represented by a series of short members. Since all combinations of node connectivity are considered, $m=n(n-1) / 2$. 


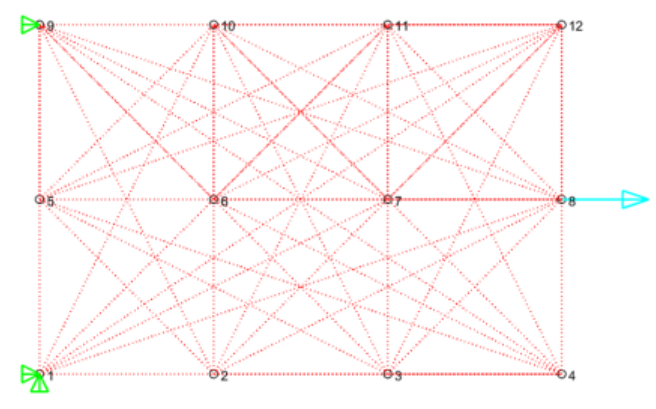

(a) Ground Structure

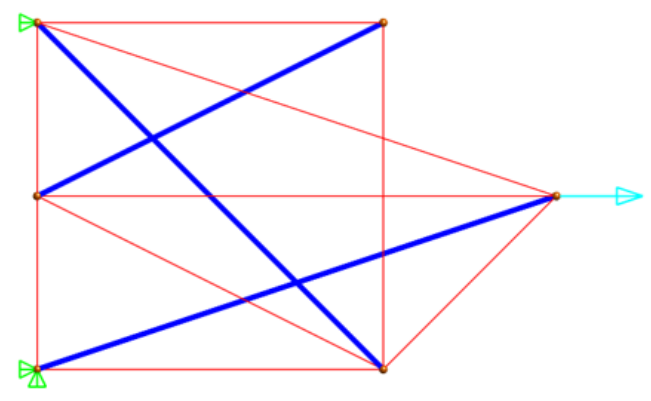

(b) Optimized solution with struts (shown in blue) and cables (shown in red)

Figure 3.1: Example of 2D tensegrity topology optimization

Figure 3.1a shows a tensegrity optimization ground structure, where a force shown in blue is applied and three green arrows represent the translational boundary conditions applied. Dashed red lines represent each potential member allowed in the optimization. Figure $3.1 \mathrm{~b}$ showcases an optimal solution to the problem posed in Figure 3.1a. In a tensegrity system, it is common to use the same strut cross-section area $a_{s}$, material density $\rho_{s}$, and elastic modulus $Y_{s}$. Similarly for all cables: $a_{c}, \rho_{c}$, and $Y_{c}$. These properties are specified prior to the optimization.

\subsection{Integers to Represent Candidate Member State}

The primary task in the tensegrity topology optimization is to partition the set of candidate members $\mathbb{M}$ into disjoint subsets containing struts $\mathbb{S}$, cables $\mathbb{C}$, and eliminated members $\mathbb{E}$. Thus, $\mathbb{S} \cap \mathbb{C} \cap \mathbb{E}=\mathbb{M}$. To accomplish the partitioning of candidate member $k$, 
variables $s_{k}$ and $c_{k}$ are defined as originally proposed by Kanno [7],

$$
\begin{aligned}
& \left(s_{k}, c_{k}\right)=(1,0) \Leftrightarrow k \in \mathbb{S} \\
& \left(s_{k}, c_{k}\right)=(0,1) \Leftrightarrow k \in \mathbb{C} \\
& \left(s_{k}, c_{k}\right)=(0,0) \Leftrightarrow k \in \mathbb{E}
\end{aligned}
$$

Vectors $\mathbf{s}=s_{k} \in \mathbb{R}^{\mathrm{m} \times 1}$ and $\mathbf{c}=c_{k} \in \mathbb{R}^{\mathrm{m} \times 1}$ are formed to denote the state of all candidate members. An additional binary variable $b_{k}$ is defined when $k \in \mathbb{C}$, which serves as an indicator when representing whether member $k$ is taut $\left(b_{k}=1\right)$ or slack $\left(b_{k}=0\right)$. Vector $\mathbf{b}=b_{k} \in \mathbb{R}^{\mathrm{m} \times 1}$ is formed.

\subsection{Objective}

Many alternative objectives are proposed in the literature including minimizing the number of cables, the number of strut length types, the differences in cable forces, etc. [37]. Consistent with most mechanical design problems, the optimization objective in this work is to minimize the mass of the tensegrity system,

$$
\min _{\mathbf{s}, \mathbf{c}, \mathbf{b}, \mathbf{t}_{0}, \mathbf{t}, \mathbf{e}_{s}, \mathbf{e}_{c}, \mathbf{d}}\left\{\left(a_{s} \rho_{s}\right) \mathbf{l}^{T} \mathbf{s}+\left(a_{c} \rho_{c}\right) \mathbf{l}^{T} \mathbf{c}\right\}
$$

\subsection{Constraints}

The optimization is constrained by the following conditions, all of which are formulated to be linear in the design variables.

1. Candidate state: Each candidate member must be either a strut, cable or eliminated.

$$
s_{k}+c_{k} \leq 1, \quad \forall k \in \mathbb{M}
$$


2. Node connectivity: A tensegrity system should not have a node that only contains cables. Thus, if there is at least one member connected at node $i$, then there should exist a strut connecting to $i$. Let $\mathbb{N}$ be the set of candidate nodes and designating the set of members connecting to node $i$ as $\mathbb{M}_{i} \subset \mathbb{M}$,

$$
c_{k} \leq \sum_{k^{\prime} \in \mathbb{M}_{i}} s_{k^{\prime}}, \quad \forall k \in \mathbb{M}_{i}, \quad \forall i \in \mathbb{N}
$$

3. Class- $m^{c}$ tensegrity: A maximum of $m^{c}$ struts can be connected at each node, which is governed by

$$
\sum_{k \in \mathbb{M}_{i}} s_{k} \leq m^{c}, \quad \forall i \in \mathbb{N}
$$

4. Pre-tension equilibrium: $\mathbf{t}_{0}$ must satisfy Eq. (2.8). While this constraint assures that the tensegrity system is in self-equilibrium, it does not guarantee stability. The tensegrity resulting from the topology optimization is usually stable, yet a post-processing step is necessary to confirm stability through Eq. (2.22).

5. Node restraints: To withstand an external load, the tensegrity system must contain pin supports to prevent rigid-body motion. Consider $\mathbb{J}$ as the set of indices of all nodal degrees of all freedom. The set of nodal displacements that are restrained is $\mathbb{J}_{R}$, and $\mathbb{J}_{F}$ is the set of nodal degrees of freedom that may encounter displacement. Accordingly, $\mathbb{J}=\mathbb{J}_{R} \cup \mathbb{J}_{F}$. In this work, all restraints are 0 ,

$$
d_{j}=0, \quad \forall j \in \mathbb{J}_{R}
$$

6. Equilibrium with external load: t must satisfy Eq. (2.6).

7. Constitutive law: d must satisfy Eq. (2.18). However, Eq. (2.18) does not represent a linear constraint as $\mathbf{K}$ depends on $\mathbf{s}$, $\mathbf{c}$, and $\mathbf{t}_{0}$. The approach formulated by Kanno [7] 
introduces $\mathbf{e}_{s}=\left\{e_{s_{1}}, \ldots, e_{s_{m}}\right\}^{T}$ and $\mathbf{e}_{c}=\left\{e_{c_{1}}, \ldots, e_{c_{m}}\right\}^{T}$ as design variables to maintain linearity. Non-zero elongations are permitted depending on the state of member $k$ by using the linear constraints,

$$
\begin{aligned}
& \lambda s_{k} \geq-e_{s_{k}} \geq 0 \\
& \lambda c_{k} \geq e_{c_{k}} \geq 0, \quad \forall k \in \mathbb{M},
\end{aligned}
$$

where $\lambda$ is a sufficiently large constant. Using Eq. (2.19), member elongations $\mathbf{e}_{s}$ and $\mathbf{e}_{c}$ are consistent with nodal displacements $\mathbf{d}$ by using the linear constraints,

$$
\begin{aligned}
& \lambda\left(1-s_{k}\right) \geq\left|e_{s_{k}}-A_{k}^{T} \mathbf{d}\right|, \\
& \lambda\left(1-c_{k}\right) \geq\left|e_{c_{k}}-A_{k}^{T} \mathbf{d}\right|, \quad \forall k \in \mathbb{M},
\end{aligned}
$$

where $A_{k}^{T}$ is the $k^{\text {th }}$ row of $\mathbf{A}^{T}$ and $|\zeta|$ denotes the absolute value. Finally, the constitutive law is linearized with

$$
t_{k}=\bar{k}_{s_{k}} e_{s_{k}}+\bar{k}_{c_{k}} e_{c_{k}}, \quad \forall k \in \mathbb{M} \text {. }
$$

where constant elastic stiffnesses are $\bar{k}_{s_{k}}=Y_{s} a_{s} / l_{k}$ and $\bar{k}_{c_{k}}=Y_{c} a_{c} / l_{k}$.

8. Compliance: A compliance constraint requires the strain energy in Eq. 2.21 must be less than designated limit $\mathfrak{U}^{u b}$,

$$
\mathcal{u} \leq \mathcal{U}^{u b}
$$

9. Strut stress limits: The stress in the struts, with and without externally applied loads, must be within a stress limit $\sigma_{s}^{u b}$. Writing the stress constraint in terms of forces,

$$
\begin{aligned}
& a_{s} \sigma_{s}^{u b} \leq t_{0_{k}} \leq 0, \\
& a_{s} \sigma_{s}^{u b} \leq t_{k} \leq 0, \quad \forall k \in \mathbb{S} .
\end{aligned}
$$


10. Cable stress limits: The stress in the cables, with and without external loads, must be within a stress limit $\sigma_{c}^{u b}$. As with struts, the cable stress constraint is written in terms of forces,

$$
\begin{aligned}
& 0 \leq t_{0_{k}} \leq a_{c} \sigma_{c}^{u b}, \\
& 0 \leq t_{k} \leq a_{c} \sigma_{c}^{u b}, \quad \forall k \in \mathbb{C} .
\end{aligned}
$$

11. Cables remain in tension: Member $k$ becomes slack if the elongation $e_{k}$ is negative and taut if $e_{k} \geq 0$. Thus, a cable will remain taut with the following constraints,

$$
\begin{aligned}
& 0 \leq\left(e_{c_{k}}-e_{k}\right) \leq \lambda\left(1-b_{k}\right), \\
& 0 \leq t_{k} \leq \lambda b_{k}, \quad \forall k \in \mathbb{C} .
\end{aligned}
$$

12. Intersecting members: A ground structure will contain many candidate members that intersect. Let $\mathbb{M}^{c}$ represent the set of pairs of intersecting members, such that $\left(k, k^{\prime}\right) \in$ $\mathbb{M}^{c}$ if member $k$ and member $k^{\prime}$ intersect. Only one of each intersecting set of candidate members can be a strut or cable,

$$
s_{k}+s_{k^{\prime}}+c_{k}+c_{k^{\prime}} \leq 1 \quad \forall\left(k, k^{\prime}\right) \in \mathbb{M}^{c} .
$$

13. Control nodes: $\mathrm{Xu}$ et al. [37] introduced a condition to include nodes at critical positions to control the overall shape of the optimized tensegrity system. As a less complicated alternative to Xu's approach, a node will be included in the optimized system if at least one strut is connected to that node and all prior constraints are enforced. Thus,

$$
\sum_{k \in \mathbb{M}_{i}} s_{k} \geq 1, \quad \forall i \in \mathbb{N}
$$

14. Non-coplanar members: $\mathrm{Xu}$ et al. [36] includes a constraint to ensure all members connecting to a node cannot lie in the same plane. This ensures a node always has 
stiffness in three directions, reducing mechanisms which lead to numerical instability. Let $P_{i}$ be a label set of coplanar members connecting to node $i$ and $\alpha_{i}^{p}$ be the coplanar member set in the $p$ th plane for node $i$.

$$
\sum_{k \in \alpha_{i}^{p}}\left(s_{k}+c_{k}\right)+\frac{\sum_{k \in \mathbb{M}_{i}}\left(s_{k}+c_{k}\right)}{\left|\mathbb{M}_{i}\right|} \leq \sum_{k \in \mathbb{M}_{i}}\left(s_{k}+c_{k}\right)\left(\forall p \in P_{i}\right), \quad \forall i \in \mathbb{N}
$$

With the objective and constraints being linear in the binary variables $\mathbf{s}, \mathbf{c}, \mathbf{b}$, and continuous variables $\mathbf{t}^{0}, \mathbf{t}, \mathbf{e}_{s}, \mathbf{e}_{c}, \mathbf{d}$, the optimization is posed as a mixed-integer linear programming problem. Hence, it can be solved globally with a branch-and-cut algorithm [35]. The algorithm works by first finding a starting feasible solution using a heuristic, often by solving the problem with no integer constraints as a linear problem. A search tree is developed outward from the starting feasible solution where the algorithm splits the problem into smaller search points or branches while trying to minimize the objective function. The algorithm sometimes determines a search branch has become unfeasible due to constraint bounds and as such the branch is cut so that the algorithm doesn't waste time searching it. Since the algorithm accounts for every possible solution, either by applying it or blanket deeming it infeasible, a global optimal solution is eventually found and proved. After conducting the optimization, Eq. (2.22) must be verified to ensure the tensegrity system is stable. 


\section{CHAPTER IV}

\section{METHODS OF ANALYSIS FOR WING COMPARISON STUDY}

To facilitate the comparison of the tensegrity-based wing structures in this work with a conventional baseline wing, it was necessary to formulate skin elements capable of integrating in the tensegrity analysis, with the skin elements roughly equivalent to those used for the analysis of the conventional wing. In addition, it was necessary to develop a consistent set of aerodynamic loading conditions that could be applied to each wing design, with or without the external skin. These components of the present study are described in the following subsections.

\subsection{Skin Elements}

A custom finite element skin code was written in MATLAB to directly interface with the tensegrity analysis codes. In particular, the appropriately-transformed element stiffness matrices of the skin elements were added to Eq. (2.18). The element type for the skin was chosen to be a superposition of the discrete Kirchhoff theory (DKT) element [3], which provides bending stiffness in the out-of-plane translational and rotational degrees-of-freedom, and the constant strain triangular (CST) element [18], which provides membrane-like stiffness for the in-plane translational degrees-of-freedom. Basic comparisons of this DKT-CST element were conducted in which it was compared with the closest corresponding element type in Abaqus, STRI3, which was used in the analysis of the baseline conventional wing skin in Section VII. In one-, two-, and four-element systems with in-plane and out-of-plane loading (and including coplanar and skew systems), the results were observed to lie within $1 \%$ of those generated in Abaqus, which was deemed an acceptable accuracy for the present study. 


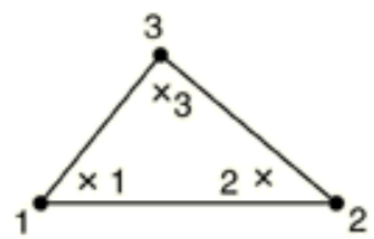

Figure 4.1: Abaqus STRI3 element with integration points

\subsection{Aerodynamic Loading Conditions}

Two aerodynamic load cases were selected based on the outer-mold-line and gross weight of the baseline conventional design, selected as the Van's RV-4, a small general aviation manned aircraft. Namely, a $2 \mathrm{~g}$ pullup maneuver was considered as a sizing case, and a $1 \mathrm{~g}$ pushover was included to interrogate the robustness of the asymmetrical airfoil geometry to downward loading. Since tensegrity systems consist of tension-only and compression-only members, the structural response may be quite different with reversed loading, which could be exploited by the pushover case.

The aerodynamic pressure loads were generated using the XFOIL Subsonic Airfoil Development System program [22], a widely-used solver that simulates viscous (or inviscid) flow over isolated 2D airfoil sections, for the constant NACA 23015 airfoil. For the load generation, a maneuvering speed of $71 \mathrm{~m} / \mathrm{s}(159 \mathrm{mph})$ was assumed (based on performance specifications for aircraft similar to the RV-4 [8]), the angle-of-attack was set at $5^{\circ}$, and standard atmospheric conditions at sea level were used, which gives a kinematic viscosity of $1.460 \times 10^{-5} \mathrm{~m}^{2} / \mathrm{s}[9]$. With the $1.47 \mathrm{~m}(4.82 \mathrm{ft})$ chord, the Reynold's number can be computed as $7.15 \times 10^{6}$, which approaches inviscid flow. An elliptical spanwise pressure distribution was assumed to distribute the XFOIL pressure loads along the wing, and the pressure distributions were scaled for the $+2 \mathrm{~g}$ and $-1 \mathrm{~g}$ load cases with the assumption that 
each wing supports half of the $680 \mathrm{~kg}(1500 \mathrm{lb}) \mathrm{RV}-4$ gross weight (multiplied by the load factor); these low-order aerodynamic assumptions were deemed sufficient for the fidelity required in the present structural study.

\subsection{Load Interpolation to Structural Nodes}

Since this work is concerned with the structural performance of tensegrity-based internal wing structures, both with and without external skins, a method was developed to interpolate the aerodynamic pressure loads to the internal structural nodes. The pressures generated in XFOIL and scaled appropriately for the elliptical spanwise distribution were converted to point forces using the respective areas over which they act, with each force placed at the centroid of the trapezoidal pressure region. Next, each vertical force $(y$ direction force in Figure 4.2) was distributed spanwise to the two bounding structural span stations (i.e., ribs in the case of a conventional wing) such that the force and the moment about the $x$-axis are each preserved. The force was then distributed chordwise to the adjacent structural nodes, again to preserve the force and now the moment about the $z$-axis. Consequently, referring to the diagram in Figure 4.2, the $y$-direction force on each of the four internal structural nodes around an aerodynamic force element $f_{y}$ can be expressed as:

$$
\begin{aligned}
& f_{1 y}=\left(\frac{\gamma_{z}}{\beta_{z}+\gamma_{z}}\right)\left(\frac{\gamma_{x}}{\beta_{x}+\gamma_{x}}\right) f_{y}, \quad f_{2 y}=\left(\frac{\gamma_{z}}{\beta_{z}+\gamma_{z}}\right)\left(\frac{\beta_{x}}{\beta_{x}+\gamma_{x}}\right) f_{y} \\
& f_{3 y}=\left(\frac{\beta_{z}}{\beta_{z}+\gamma_{z}}\right)\left(\frac{\gamma_{x}}{\beta_{x}+\gamma_{x}}\right) f_{y}, \quad f_{4 y}=\left(\frac{\beta_{z}}{\beta_{z}+\gamma_{z}}\right)\left(\frac{\beta_{x}}{\beta_{x}+\gamma_{x}}\right) f_{y} .
\end{aligned}
$$

Similar equations can be written for the pressure-derived $x$-direction forces, but using the relative distances in the $y$-direction in place of $\beta_{x}$ and $\gamma_{x}$ in Figure 4.2. This approach amounts to a splining method that distributes the aerodynamic forces according to 
their individual action on each of the neighboring structural grid points, permitting small membrane-like deformation of the skin with pinned connections at the ribs.

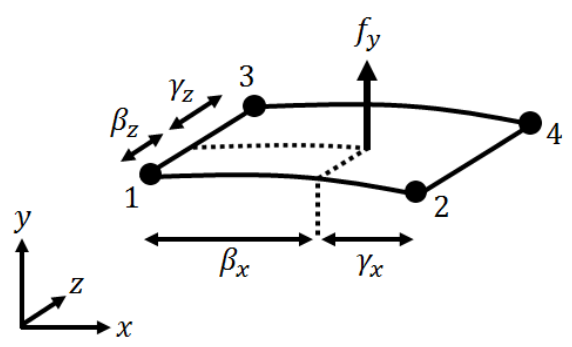

Figure 4.2: A diagram showing an aerodynamic force element $f_{y}$ in the $y$-direction, at an arbitrary location on the wing surface, and the four closest internal structural nodes, labeled $1,2,3$, and 4 . The dimensions $\beta_{z}$ and $\gamma_{z}$ are measured along the $z$-direction, and the dimensions $\beta_{x}$ and $\gamma_{x}$ are measured along the $x$-direction. 


\section{CHAPTER V}

\section{WING DESIGNS}

\subsection{Baseline Conventional Wing}

As aforementioned, the Van's RV-4 [31, 33], a manned aircraft produced by Van's Aircraft and sold as a kit, was selected as the baseline aircraft for the present structural comparison study. The RV-4 is similar in size and weight to the class of Unmanned Aerial Vehicles (UAVs) that are of interest in this study, given the suitability of UAVs for morphing capabilities that tensegrity-based designs may enable. In addition, much of the structural design data could be obtained for the RV-4 from open literature [31, 33], unlike most commercial and military aircraft; moreover, parameters which were not readily available were simply estimated in an attempt to construct a model reasonably representative of the RV-4.

The Van's RV-4 aircraft has a $7.0 \mathrm{~m}$ (23 ft) wingspan and a gross weight of approximately $680 \mathrm{~kg}(1500 \mathrm{lb})[14,31,33]$. The wings use a constant airfoil shape, the NACA 23015 airfoil, with no sweep or taper, and a chord length of $1.47 \mathrm{~m}$ (4.82 ft). The wing structure consists of 14 ribs, non-uniformly spaced, and 2 spars within the wing box section, as well as 12 ribs in the flap section. The ribs and spars are made of aluminum alloy 2024-T3, and the skin of aluminum 2024-T4, each with a Young's modulus of 73 GPa (10.6 Mpsi), a Poisson's ratio of 0.33 , and a density of $2780 \mathrm{~kg} / \mathrm{m}^{3}\left(0.1 \mathrm{lb} / \mathrm{in}^{3}\right)$ [13]. With these material properties, a finite element model was constructed in Abaqus FEA to represent the wing section from the root to the beginning of the wingtip closeout, which encompasses a span length of $2.81 \mathrm{~m}$ (110.75 in). The ribs and spars were modeled as thin, 2D sections consisting of the webs and flanges, with thicknesses ranging from $0.64 \mathrm{~mm}(0.025 \mathrm{in})$ to $1.02 \mathrm{~mm}(0.040 \mathrm{in})[14,31,33]$. The I-beam cross-sections assigned to the spars were intended to be more representative of 
the stiffness and mass properties than rectangular cross-sections, attributable in part to the rib-spar connection structures that were not explicitly modeled. Finally, the skin structure of thickness $0.64 \mathrm{~mm}(0.025 \mathrm{in})$ was connected to the ribs at the exterior rib flanges. Figure 5.1 shows the Abaqus model with the skin removed and then included. The mass of the structure is $10.8 \mathrm{~kg}(23.9 \mathrm{lb})$ without the skin, and $25.7 \mathrm{~kg}(56.6 \mathrm{lb})$ with the skin.

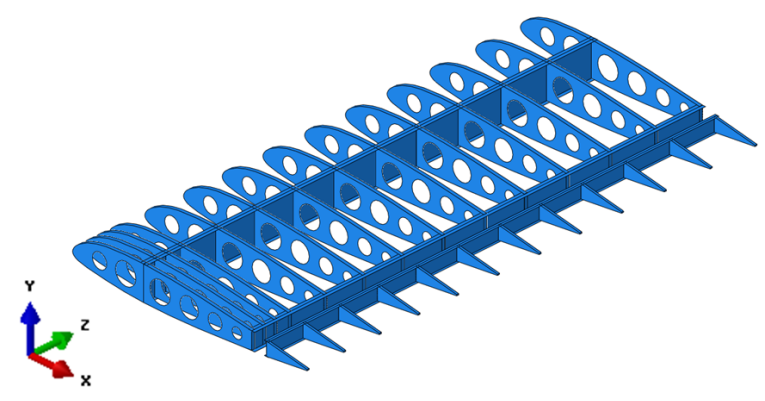

(a)

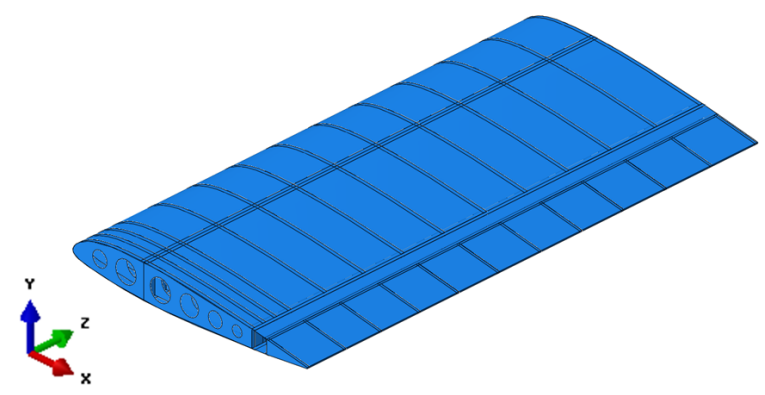

(b)

Figure 5.1: The conventional wing structure in Abaqus FEA, constructed to be representative of the notional RV-4 aircraft wing structure, for the models a with no skin and b with the skin.

\subsection{Appropriate Nodal Locations for Tensegrity Wing Designs}

In constructing tensegrity-based wing designs, a ground structure of nodes (nodal locations) had to first be defined to allow for combinations of element connectivities. As such, 
an intermediate optimization was conducted to determine the ideal location for 10 nodes to be placed around the perimeter of the NACA 23015 airfoil of chord length $1.47 \mathrm{~m}$ (57.87 in). The optimization objective was to minimize the distance between the piecewise linear curve defined by the nodes and the airfoil shape that connects the nodes. Figure 5.2 illustrates the location of the 10 nodes on the airfoil that resulted from the optimization, wherein each pair of nodes on the top and bottom surfaces were required to lie at the same chordwise position. These locations serve as key parameters for the two tensegrity wings explored in the following sections.

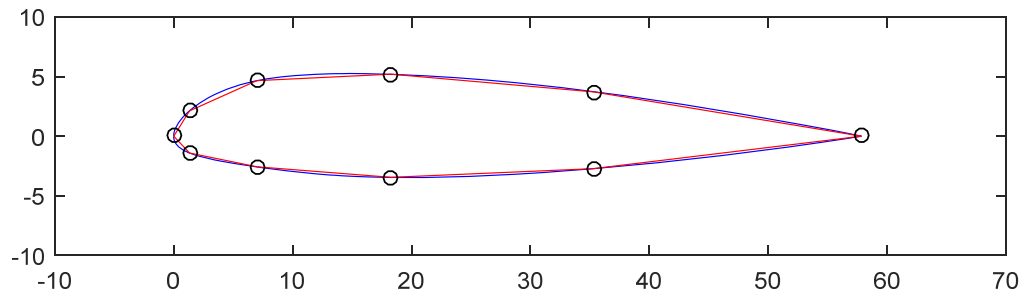

Figure 5.2: Optimal location for 10 nodes to approximate a NACA 23015 airfoil.

The spanwise spacing of the nodes was set by the need to match the structural stiffness observed in the RV-4 model (see also Sect. VII). In particular, a finite element analysis of a portion of the skin reveals that a maximum unsupported length of $355 \mathrm{~mm}$ (14 in) near the high-pressure leading edge is required to conform to the stiffness observed for the RV-4. It should also be noted that the same skin material and thickness were used for the tensegrity wing analysis as for the conventional wing (aluminum 2024-T4 with thickness $0.635 \mathrm{~mm}$ $(0.025$ in $))$. 


\subsection{Tensegrity Wing Developed through Designer Insights}

The first tensegrity wing design in this study was developed based on the properties of individual tensegrity unit cells, and with standard design practices employed. Namely, in a prior study on the stiffness and strength of various tensegrity unit cells [19], the R-cross (in Figure 2.1) was ranked among the most structurally efficient, exhibiting high stiffness and high strength for a given weight. Additionally, the rectangular structure allows it to be systematically combined to create large composite structures.

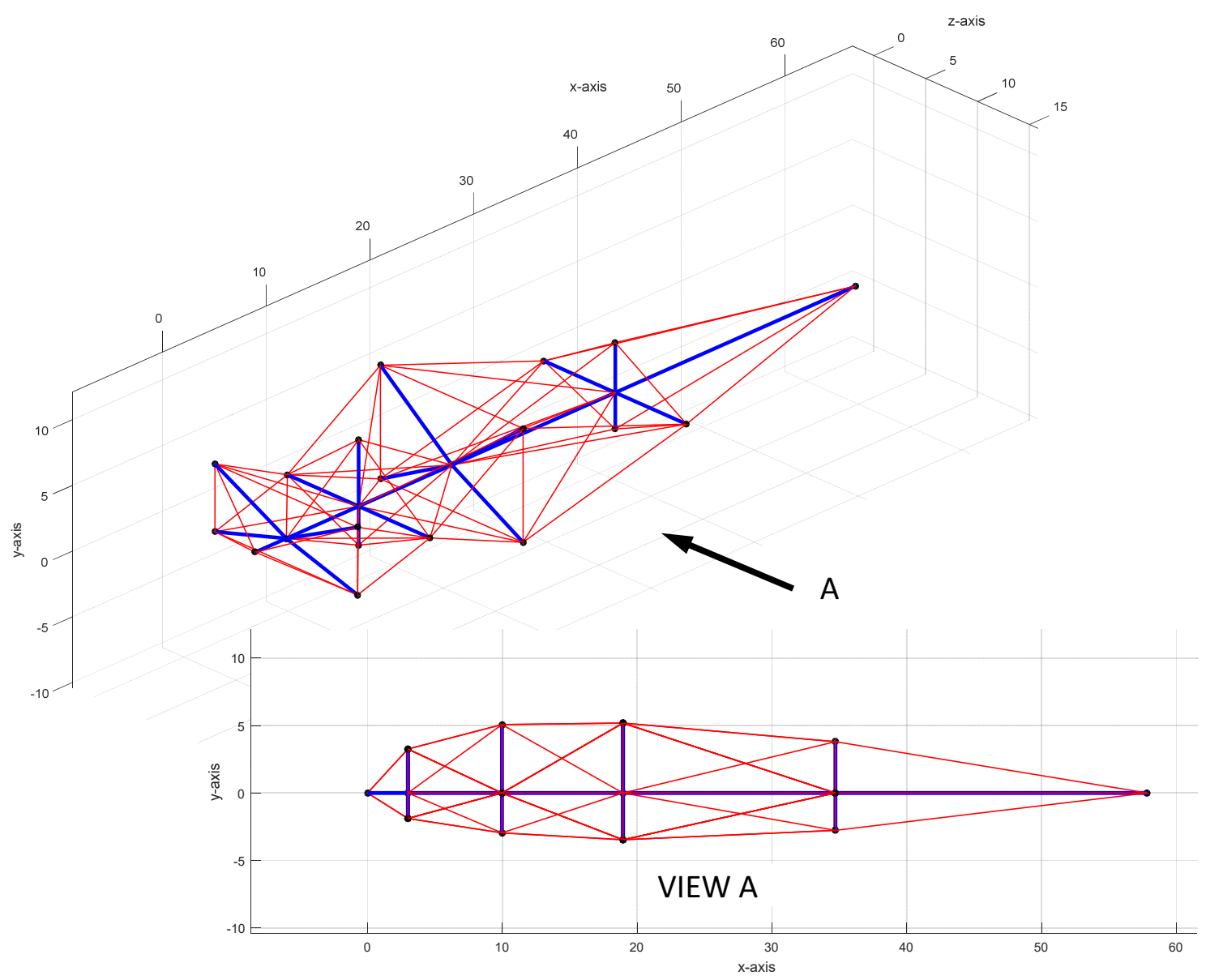

Figure 5.3: Four R-cross unit cells are combined to form a tensegrity rib-like structure. Struts are shown in blue and cables are red. 
Using engineering design insights, four rectangular cross unit cells are assembled into a rib-like structure shown in Figure 5.3. Note that each of the four R-cross cells are staggered at $45^{\circ}$ increments. The length of the $m_{s}=21$ struts are selected to locate nodes in the optimal positions that approximate the NACA 23015 as determined in Sect. 5.2. The width of the rib-like segment is $0.35 \mathrm{~m}$ (13.84 in), which corresponds to one-eighth of the 2.82 $\mathrm{m}$ (110.75 in) wing length and sufficiently supports the skin as stated in Sect. 5.2. The full wing dimensions are equal to those of the Van's RV-4 baseline wing. The struts are formed into a stable and self-equilibrium state by using $m_{c}=48$ cables, having $n_{s}=12$ independent pre-load modes.

A stable, full wing tensegrity structure is created by joining eight rib-like segments, spanwise, as shown in Figure 5.4. However, structural redundancy is required for flight vehicles, ensuring that the structure will not fail if some part is damaged or removed. As such, redundancy is created by using 14 additional cables to span the outer nodes and eliminate infinitesimal mechanisms. The full wing then uses $m_{s}=168$ struts and $m_{c}=398$ cables (i.e., $m=566$ ), connected at $n=134$ nodes. Notice that the tensegrity wing uses no spars and greatly relies on the outer, spanwise cables for stiffness. The struts are made from aluminum tubing with an elastic modulus of $72 \mathrm{GPa}(10.4 \mathrm{Mpsi})$ and density 2700 $\mathrm{kg} / \mathrm{m}^{2}\left(0.098 \mathrm{lb} / \mathrm{in}^{3}\right)$. For the braided steel cables, an apparent modulus of elasticity of 137 GPa (19.9 Mpsi)and a density of $6450 \mathrm{~kg} / \mathrm{m}^{2}\left(0.23 \mathrm{lb} / \mathrm{in}^{3}\right)$ was used.

With a suitable topology identified, a sizing optimization was performed on the entire wing to appropriately size the cables. To standardize the strut-to-strut connections, standard aluminum tubing with outer diameter of $11.1 \mathrm{~mm}(0.438 \mathrm{in})$ and $1.8 \mathrm{~mm}$ (0.070 in) wall thickness were used for all struts. Allowable cable diameters were taken as commerciallyavailable, $7 \times 7$ stranded aircraft cables. All nodes at the wing root were restrained against 


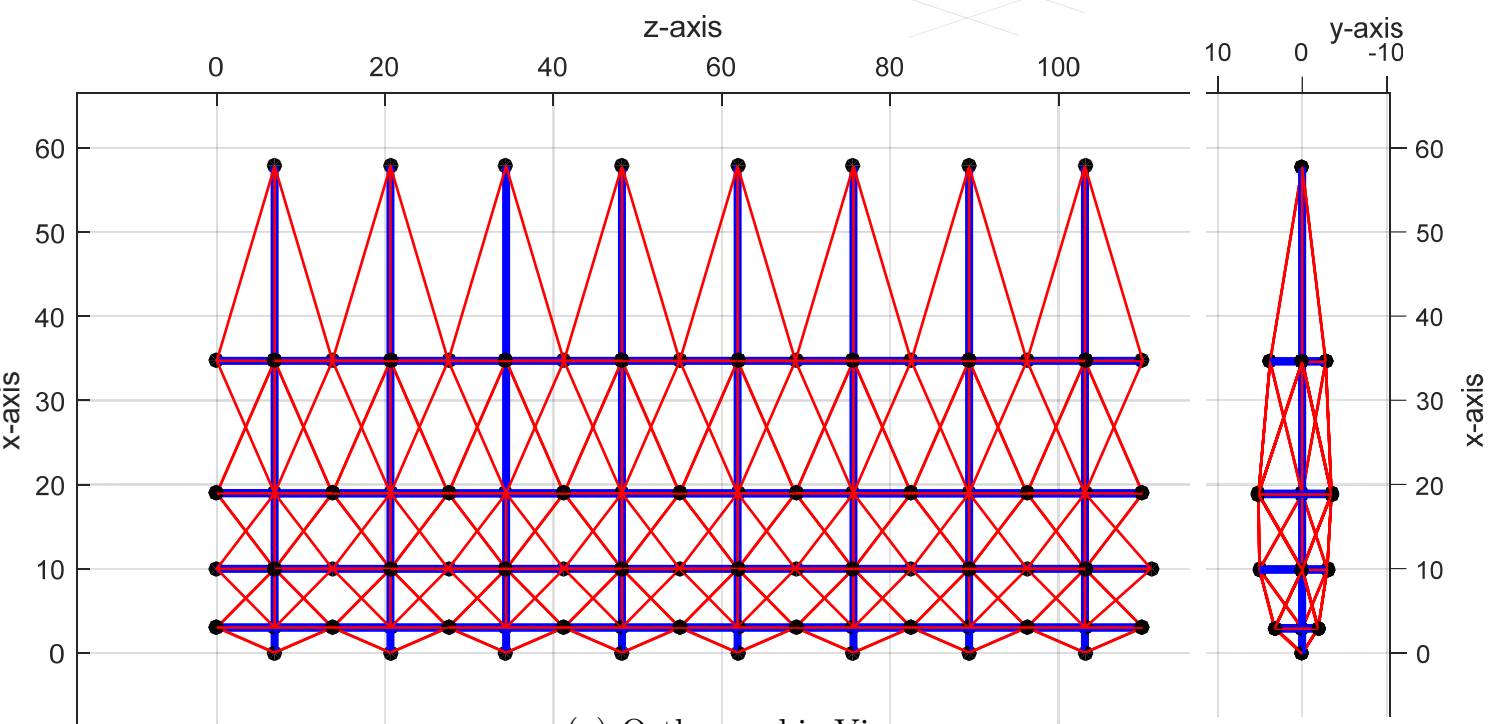

(a) Orthographic Views

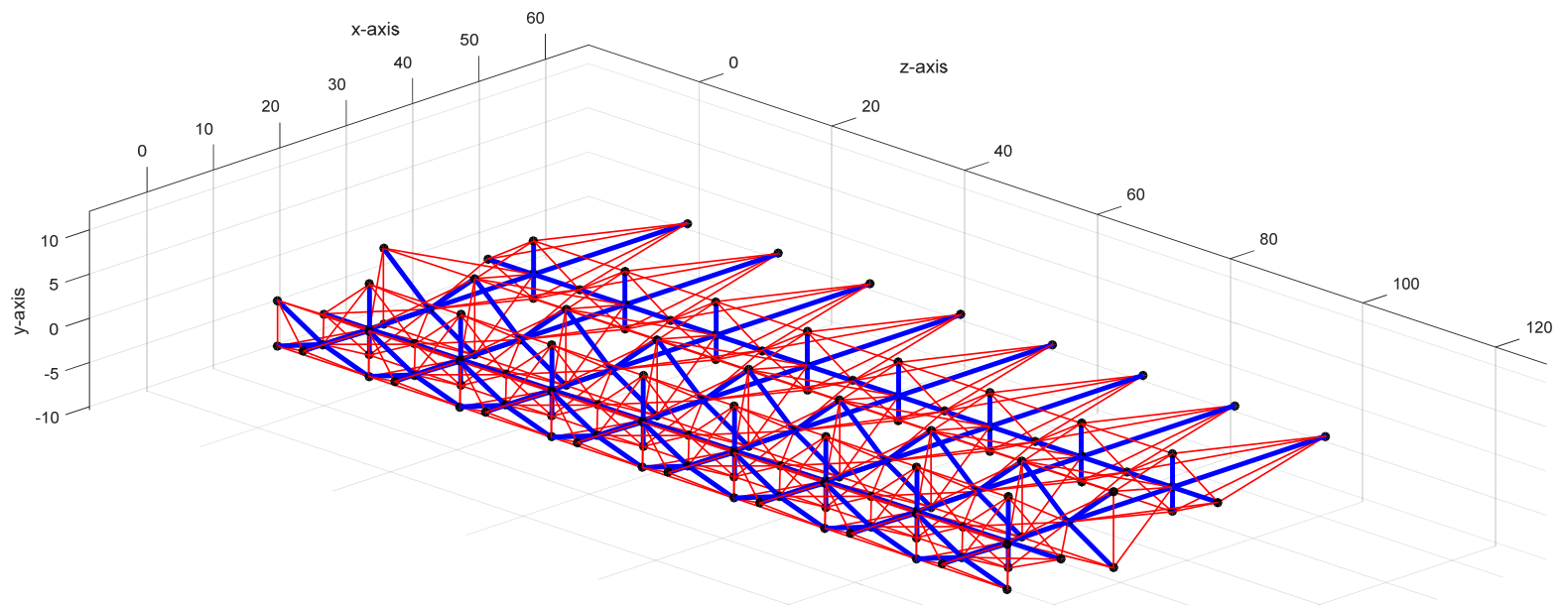

(b) Isometric View
z-axis
$80 \quad 100$
$10 \quad 0 \quad \begin{array}{r}y \text {-axis } \\ -10\end{array}$

$\begin{array}{rrrr}0 & 20 & 40 & 60\end{array}$

Figure 5.4: Eight rib-like segments of Figure 5.3 are combined to form a tensegrity wing 60 concept.

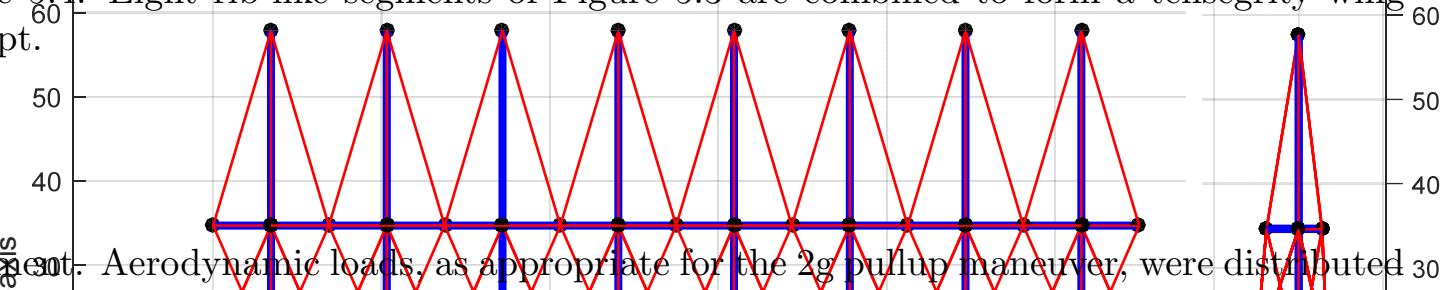

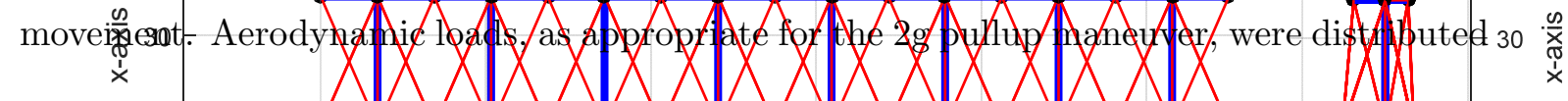
to the redal locat was milnum 20

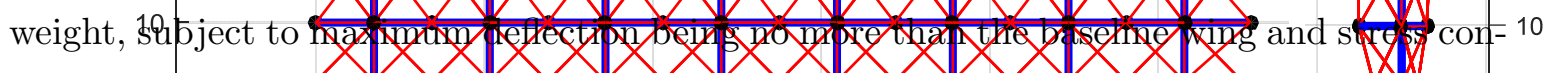

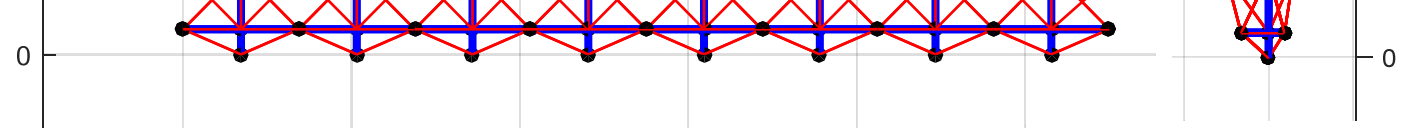


straints. Of the 398 cables, Table 5.1 provides the distribution of diameters. The resulting tensegrity wing design, without the skin, weighed $6.4 \mathrm{~kg}(14.2 \mathrm{lb})$.

Table 5.1: Cable diameters used in the designer-developed tensegrity aircraft wing.

\begin{tabular}{|r|c|c|c|c|c|c|c|c|c|c|c|}
\hline Cable Dia $(\mathrm{mm})$ & 1.00 & 2.38 & 2.78 & 3.18 & 3.57 & 3.97 & 4.37 & 4.76 & 5.16 & 5.56 & 5.95 \\
\hline Cable Dia (in) & 0.039 & $3 / 32$ & $7 / 64$ & $1 / 8$ & $9 / 64$ & $5 / 32$ & $11 / 64$ & $3 / 16$ & $13 / 64$ & $7 / 32$ & $15 / 64$ \\
\hline No of cables & 215 & 73 & 16 & 12 & 17 & 13 & 15 & 16 & 11 & 7 & 3 \\
\hline
\end{tabular}

\subsection{Tensegrity Wing Developed through Topology Optimization}

The topology optimization formulation described in Sect. III and the ground structure approach discussed in Sect. 5.2 were applied to generate a second tensegrity-based wing design for this comparison study. The node locations for the ground structure are shown in Figure 5.5a and consist of three offset layers that represent the root portion of the wing. Each layer contains a grid of 14 potential nodes that fit within the NACA 23015 airfoil. The three layers are offset $0.28 \mathrm{~m}$ (11.08 in), producing a $0.56 \mathrm{~m}(22.16 \mathrm{in})$ segment that corresponds to a one-fifth wing portion of the $2.82 \mathrm{~m}$ (110.75 in) span. Thus, the ground structure forms a rib-like segment similar to the approach used in Sect. 5.3. The one-fifth wing segment approach, using an $n_{p}=42$ node ground structure was adopted to produce an optimization that is tractable in terms of computational requirements. Potential elements were constructed with full connectivity [38]; that is, potential elements are created between all nodes. As such, the one-fifth wing segment ground structure has $m_{p}=861$ potential elements. From Eq. (3.2), each potential element has eight design variables $\left(\mathbf{s}, \mathbf{c}, \mathbf{z}, \mathbf{t}^{0}\right.$, $\left.\mathbf{t}, \mathbf{e}_{s}, \mathbf{e}_{c}, \mathbf{u}\right)$, which results in a tensegrity wing MILP optimization with 6,153 variables. Since this segment represents the portion at the wing root, all 14 nodes around the airfoil 
exterior of Layer I were restrained in all three translational directions. Lift forces were placed on eight nodes of Layers II and III as appropriate for the $2 \mathrm{~g}$ pullup aerodynamic loading condition (see Figure 5.5a). Similarly, spanwise loads were placed on four nodes of Layer III to emulate the bending moment transferred from the aerodynamic loading of the outward portion of the wing.

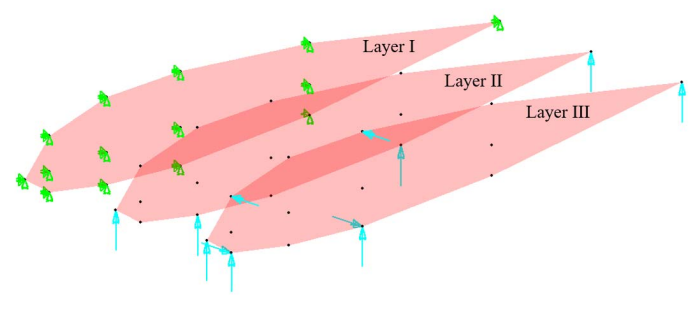

(a) Ground Structure

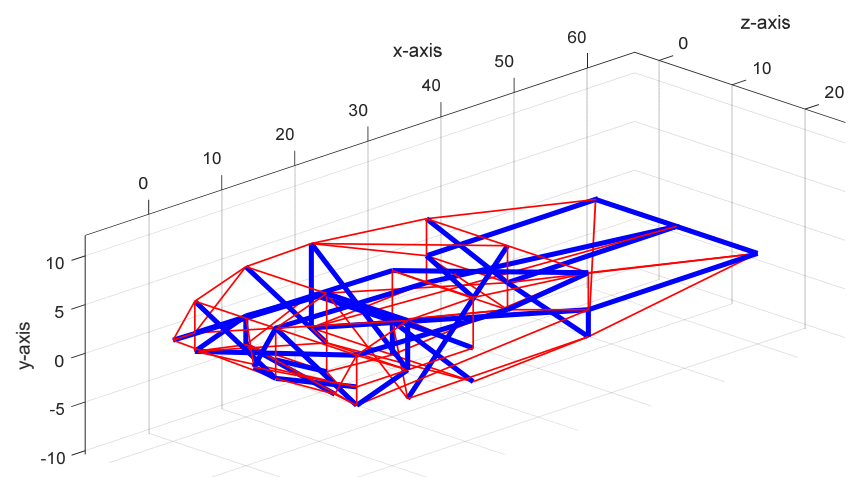

(b) Optimized Topology

Figure 5.5: Ground structure mesh for the tensegrity topology optimization of a one-fifth span wing segment and corresponding optimization result.

The topology optimization was run on the Ohio Supercomputer Center [5], using a Dell PowerEdge C6420 two-socket server with Intel Xeon Gold 6148 (Skylake, 20 cores, 2.40GHz) processors and 192GB of memory. The MILP optimization is performed using IBM ILOG CPLEX Optimization Studio 12.8.0. The optimization was stopped after 48 hours, achieving an objective value of $3.23 \mathrm{~kg}(7.18 \mathrm{lb})$. As shown in Figure $5.6 \mathrm{~b}$, the resulting one-fifth span from the topology optimization has a higher concentration of struts 
than the designer-developed wing, containing $m_{s}=40$ struts and $m_{c}=83$ cables, with $n_{s}=3$ pretension modes.

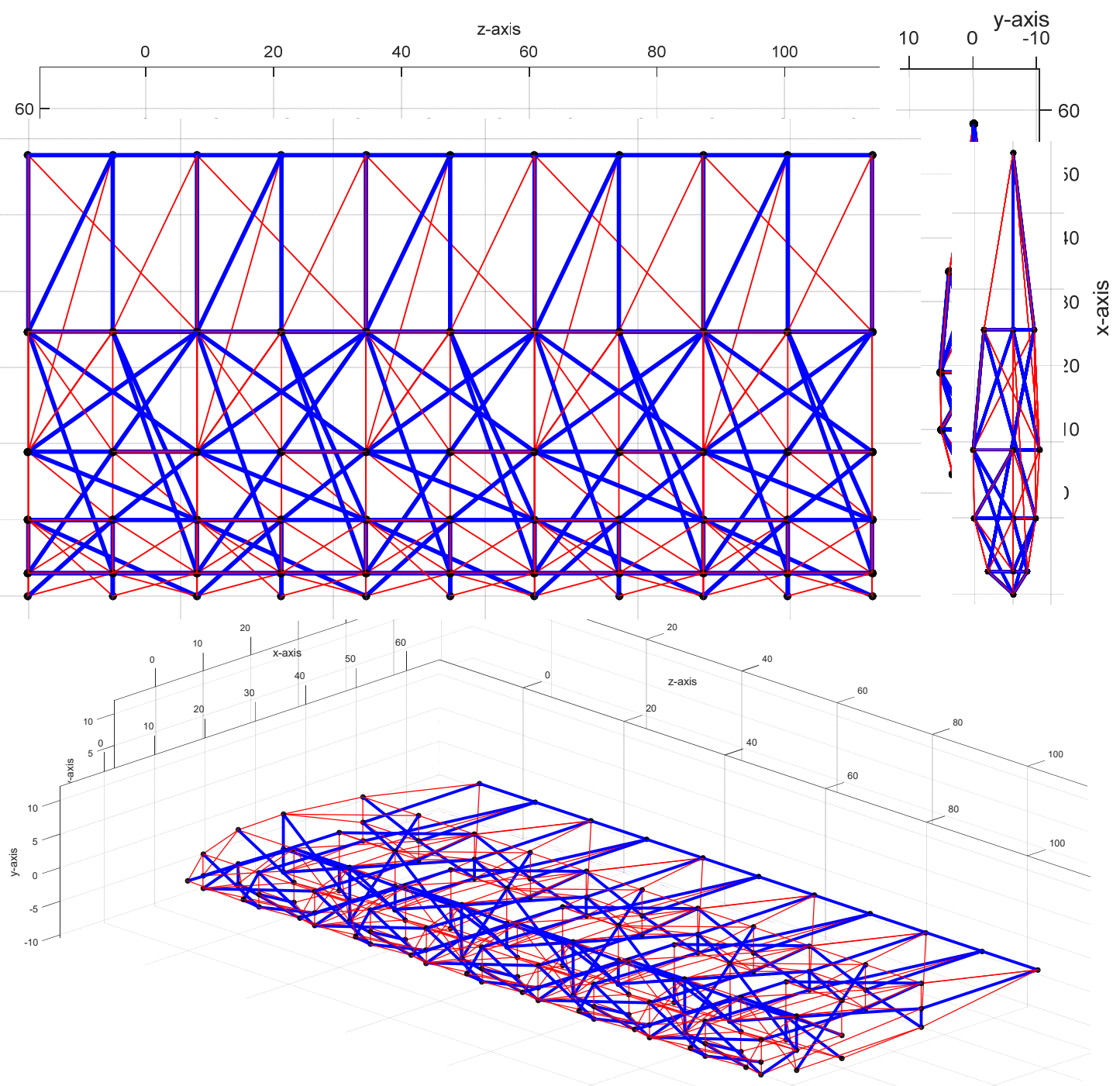

(b) Isometric View

Figure 5.6: Topology-optimized tensegrity wing concept consisting of five combined wing segments of Figure 5.5. 
After joining five segments spanwise, the resulting wing structure as shown in Figure 5.6 was obtained. A sizing optimization was performed to adjust the diameters of struts and cables and maintain a full wing deflection level that is comparable to that of the RV-4 wing. Over the entire wing, a distribution of cable diameters shown in Table 5.2 was obtained. The mass of the topology-optimized tensegrity wing, without skin, is $5.36 \mathrm{~kg}(11.6 \mathrm{lb})$.

Table 5.2: Cable diameters used in the topology-optimized tensegrity aircraft wing.

\begin{tabular}{|r|c|c|c|c|c|c|c|c|c|}
\hline Cable Dia $(\mathrm{mm})$ & 0.91 & 1.42 & 1.57 & 1.78 & 1.80 & $2.03-7.49$ & 8.05 & 8.59 & 9.07 \\
\hline Cable Dia (in) & 0.036 & 0.056 & 0.062 & 0.070 & 0.071 & $0.080-0.295$ & 0.317 & 0.338 & 0.357 \\
\hline No of cables & 3 & 1 & 1 & 1 & 341 & 21 & 1 & 1 & 1 \\
\hline
\end{tabular}




\section{CHAPTER VI}

\section{TENSEGRITY WING PROTOTYPE DESIGN AND FABRICATION}

Supplementary to the wing analysis was the mechanical design and prototype fabrication of a scaled down model of the designer-developed tensegrity wing. This fabrication offers insight into the physical challenges with building tensegrity structures. The fabrication allows verification of a theoretical model of tensegrity wing stiffness with the physical tensegrity wing stiffness. Additionally, the prototype will provide physical insights into construction and assembly challenges. Tensegrity wing constructions are rare within the literature so there are few usable references for design strategies and ideas. The constructed wing could be one of the only tensegrity wing structures ever built utilizing class 6 joints and tensioning devices, while being designed to withstand loading for future model validation purposes.

\subsection{Class 6 Joint}

The tensegrity structure should, in theory, have all struts and cables precisely converge at a node. Furthermore all struts and cables should be able to freely rotate about the node. During reviews of tensegrity literature no existing class 6 joint design was found, prompting the creation of the one used within this work. The joint design shown in Figure 6.1 was used since it allowed all struts to be in line with the node and allowed motion about the node for each strut. Negating the affects of friction within the joint, this motion is all that is needed in theory to confirm that no bending moment is transferred to the struts. The design consists of a low friction PTFE ball centered at the node that is enveloped by a shell split into two connecting pieces. Between the shell and ball lies a gap where an interfacing piece, called the rocker, serves as a method of allowing the strut to rotate about the center

of the ball. The rocker's inner surface rests against the convex surface of the ball while the 


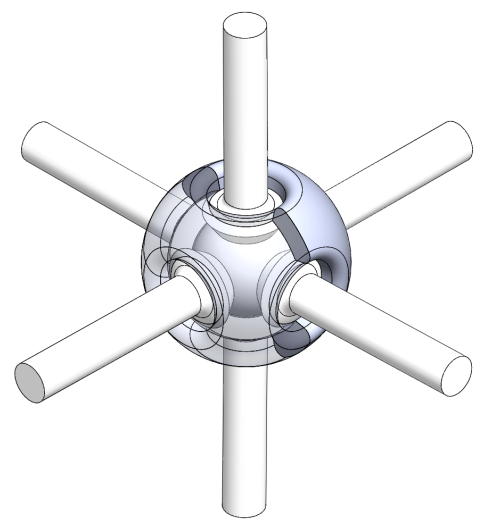

(a) Isometric View

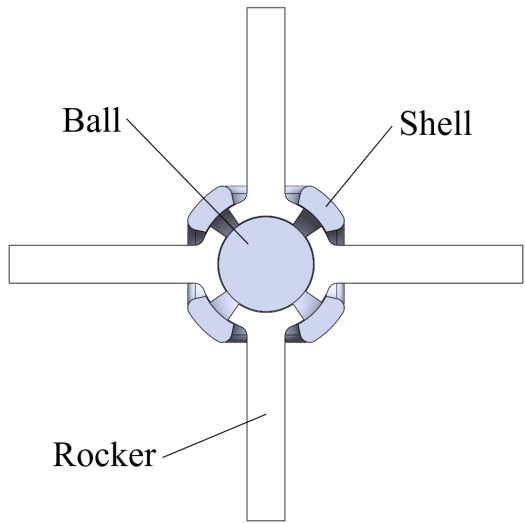

(b) Cross Section

Figure 6.1: Class 6 tensegrity joint base design

rocker's outer surface rests against the concave inner surface of the shell. During assembly four rockers are arranged between the holes split by the shell seam line with the remaining two rockers being slid into their holes prior to connecting the shell halves together.

Built upon the class 6 joint base design was the capability for cables to connect onto the joint while being aligned collinear with the node. Shown in Figure 6.2 are preliminary ideas for attaching cables to the joints. Figure 6.2a showcases a design involving a slotted rocker in which cables are routed through. The cable ends are affixed to the outer edge of the slotted rocker and an overlapping strut piece is slid onto the rocker, securing the cables in place due to the compressive nature of the strut. The design was discarded since securing the cable ends to the outer edge of the rocker would have likely been too challenging and the design makes alignment of cables towards the node difficult. Figure $6.2 \mathrm{~b}$ uses a protruding socket in which a ball end cable is inserted and pushed into a narrow slot preventing detachment. The cable socket design required an unnecessary amount of material to be an applicable 

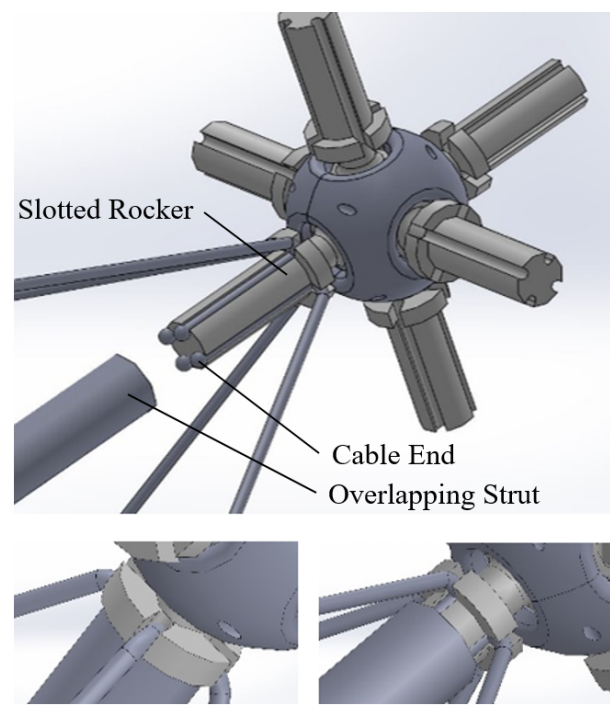

(a) Slide on struts to hold cables
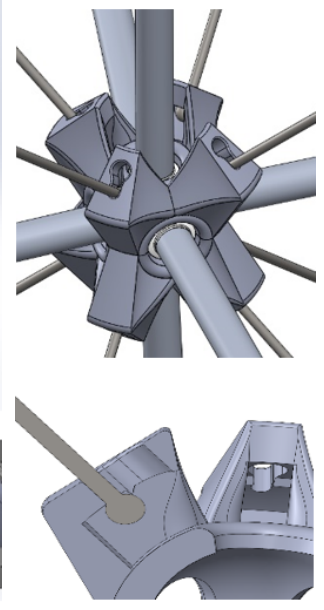

(b) Cable sockets

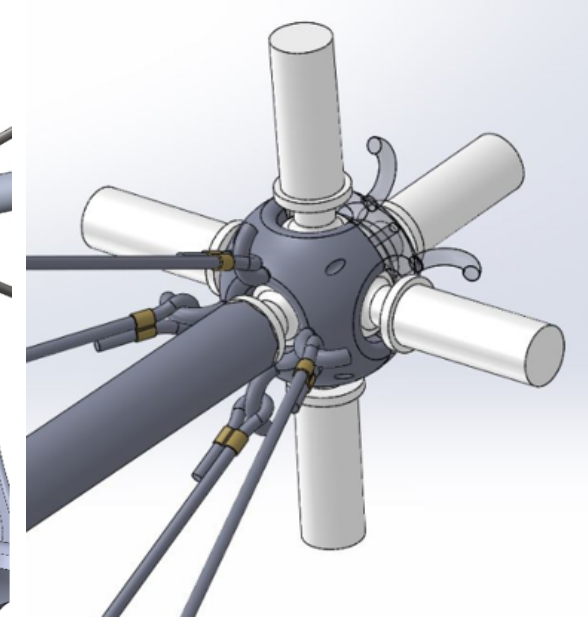

(c) Shell cable loops

Figure 6.2: Preliminary designs for joint cable connectivity

design. Figure 6.2c features loops extruded from the shell that allow the cables to hook upon them. This design proved more appealing than the previous two because it promoted easier alignment of the cables with the node, required a smaller amount of material, and allowed more accessibility for cable installation. The cable loop design eventually led to the final selected idea, which was threading miniature screw eyes into bosses with holes.

Another design considered for allowing cable connectively to the joints is shown in 6.3. This design was additively manufactured and tested since in theory it offers acceptable cable alignment with the node, uses minimal amounts of material, and serves as a way of connecting both shell halves together. The design involves metal safety wire that was wound around the shell through supported guides. The wire connects the two halves of the joint, while providing a surface for attaching cables. Although it provided a secure 

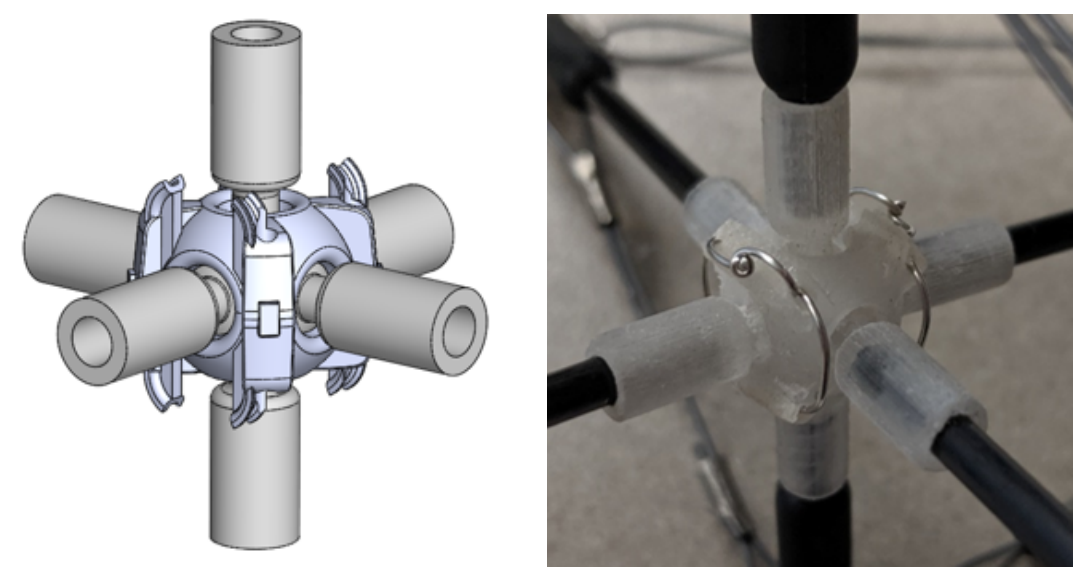

Figure 6.3: Class 6 joint design with safety wire guides

connection between the shell halves, forming the wire around the shell was difficult due to its stiffness and that the wire ends are often sharp enough to cut one's hands. As a result of the assembly difficulties this design was discarded.

Ultimately the finalized design for the class 6 joint is shown in Figure 6.4. It features screw eyes that are manually threaded into bosses extruded from the shell. The bosses are angled to prioritize the alignment of the cables with the node. The method used for connecting two shell halves involves using $1.19 \mathrm{~mm}$ (3/64") diameter acetal dowel pins that were slid within the two joint shell halves and then held in place by application of Hardman Double/Bubble ${ }^{\circledR}$ extra fast setting epoxy. The shell halves contain a connecting groove over the dowel pins that the epoxy was to be applied within. Prior to the epoxy application, the dowel pin surfaces are roughened with sandpaper and the shell halves and dowel pins are cleaned with isopropyl alcohol to promote better adhesion. The rockers contain a hole for inserting the struts, though in contrast to the basic joint design in Figure 6.1 the two 

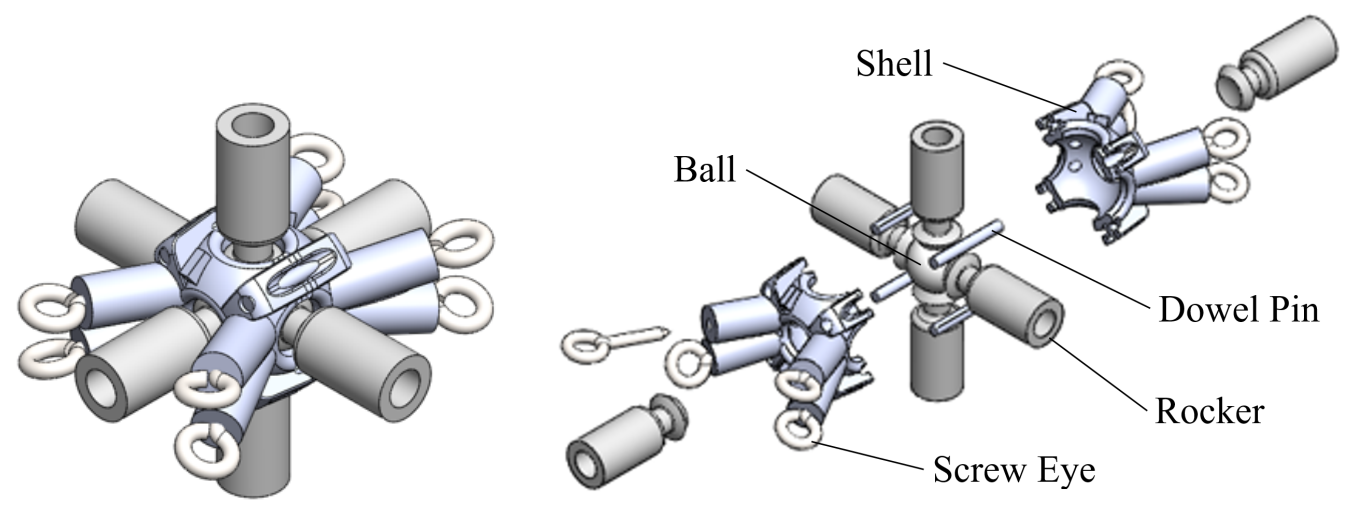

Figure 6.4: Finalized general joint design

rockers that directly insert into the shell must be forcibly pressed into the shell due to the width of the wall enveloping the strut. The ability for each cable to freely rotate about the nodes was deemed a non-feasible task. Allowing the struts rotation about the node is more critical in preventing applied bending moments at a node than the rotation of the cables. However, in the final joint design this compromise is partially relieved. The use of screw eye hooks for attaching the cables to allows minor rotation since the cables are hooked on the screw eyes, albeit the rotation is about the screw eye location instead of the nodes. In addition, due to the nature of the shell and ball design, the shell and therefore the cables can rotate about the ball as a whole.

\subsection{Class 1 Joint and Load Testing}

Nodes lying along the exterior boundary of the tensegrity structure consist of only class 1 tensegrity joints, a single strut with cables connecting towards the node. Figure 6.5 shows preliminary ideas for attaching cables to the ends of struts. Once the cable connection idea 


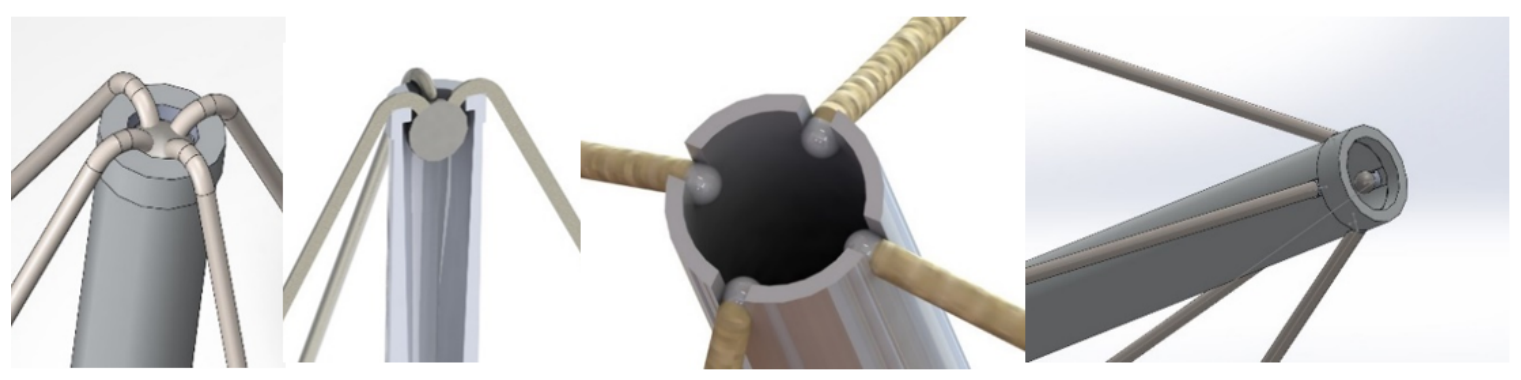

Figure 6.5: Preliminary ideas for class 1 joints

of using screw eyes in the class 6 joints was selected, using screw eyes on 3D printed strut ends was also implemented leading to the selected idea in Figure 6.6.

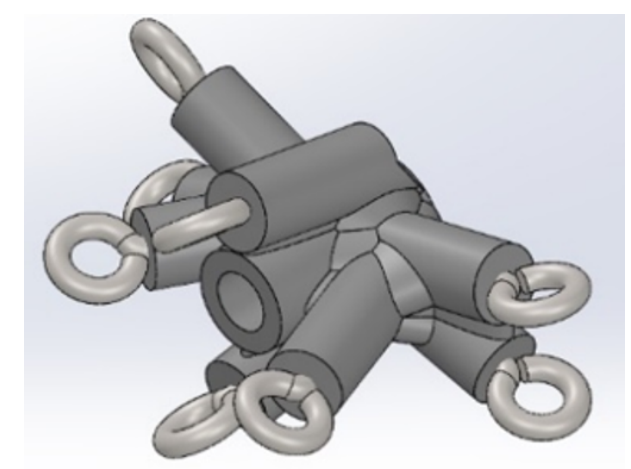

Figure 6.6: Selected idea for class 1 joints

Various load testing was done to confirm the strength of the selected designs. Figure 6.7 a displays two test pieces with bosses of differing sizes that were $3 \mathrm{D}$ printed using VeroWhitePlus ${ }^{\mathrm{TM}}$ material. The various hollow boss sizes on the side of the test piece were printed to test for fitting the $3 \mathrm{~mm}$ (0.118 in) OD, $2 \mathrm{~mm}$ (0.079 in) ID carbon fiber struts into the rockers. A $3.07 \mathrm{~mm}$ (0.121 in) rocker hole size was selected from the testing based on allowing the struts to slide into the rocker with light resistance that still retains the 
strut enough to ease the assembly process. The bosses located on the top of the test pieces were used to test the strength of the screw eye bosses as well as the screw eyes themselves. Although many different miniature screw eyes were tested the finalized ones were approximately $12 \mathrm{~mm}$ (0.47 in) long with a $5 \mathrm{~mm}$ (0.20 in) opening. The screw eyes held to an average of $106.7 \mathrm{~N}$ (24 lb) of tension force before starting to come open. These screw eyes were selected since $106.7 \mathrm{~N}$ (24 lb) was sufficient to handle the expected loading on most cables. However, a select few cables were expected to undergo forces up to $235.7 \mathrm{~N}$ (53 lb). In order to increase the strength of the screw eyes when needed, the screw eye opening seam was soldered using resin core 60/40 lead solder as shown in Figure 6.7b. Under loading the soldered screw eyes on average failed at greater than the $253.5 \mathrm{~N}$ (57 lb) limit of the Omega DFG82 force gauge used. Proper boss size selection was based upon withstanding the 235.7 N (53 lb) within the select few cables. The finalized boss size surpassed the limit of the force gauge and was $3.94 \mathrm{~mm}$ (0.155 in) OD and $1.09 \mathrm{~mm}$ (0.043 in) ID and at least 8.89 mm (0.350") long.
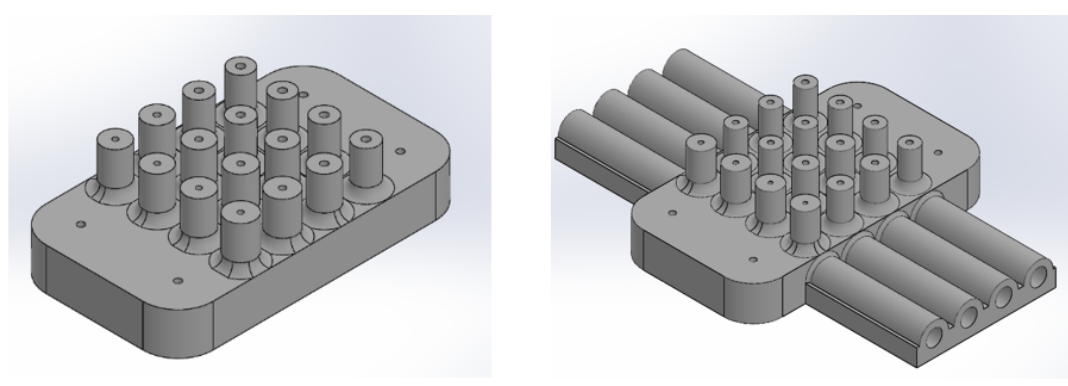

(a) Boss and strut hole sizing test pieces

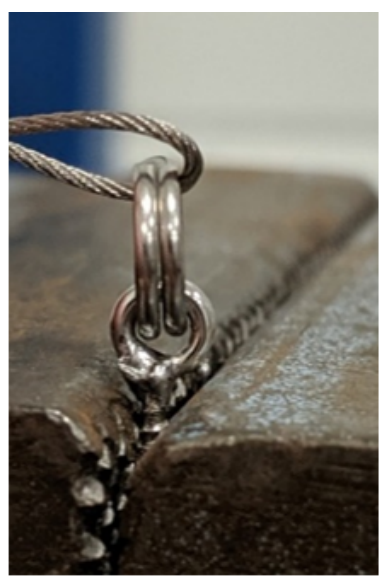

(b) Soldered screw eye

Figure 6.7: Design strength testing 


\subsection{Full Assembly}

Figure 6.8 shows the design layout of all the tensegrity wing joints and connections for a section of the wing. The design of the unique joints and connectors is based upon the finalized class 6 joint design shown in Figure 6.4 and the class 1 joint design shown in Figure 6.6. Modifications of these existing designs, accounting for cable and strut geometry, produce the unique designs encompassed in the wing section. Certain compromises were required due to the scaled down nature of the design. All cables throughout the tensegrity wing should be in-line with the node. However, since this was frequently not feasible the line of action for the cables was sometimes offset from the node.

Illustrated within Figure 6.8a are colors shown on the joint and connector bosses. Blue signifies the cable is in the line of action with the node point, as it should be. Orange signifies the cable line of action had to be offset from the node point slightly due to joint geometry. Purple bosses in Figure 6.8 indicate that the screw eye needed to hold two cables at once and the resulting boss line of action is selected to minimize the line of action offset of both cables. Whenever a cable or boss line of action was offset, the same mirrored offset was used on both sides of the wing section to ensure symmetry.

Due to the scaled down nature of the design, rockers were designed in such a way as to reduce their size such that they could still be manipulated and assembled by hand while remaining strong enough to endure expected loading. A reasonable lower size limit was reached with the rocker design shown in Figure 6.4, prompting a new required design when space restrictions arose. Figure 6.8a (right) shows two joints which have the struts intersecting the node at a narrow angle. The narrow angles forced the creation of two new joint designs. The joint shown in the upper right of Figure 6.8a uses two rockers that are 


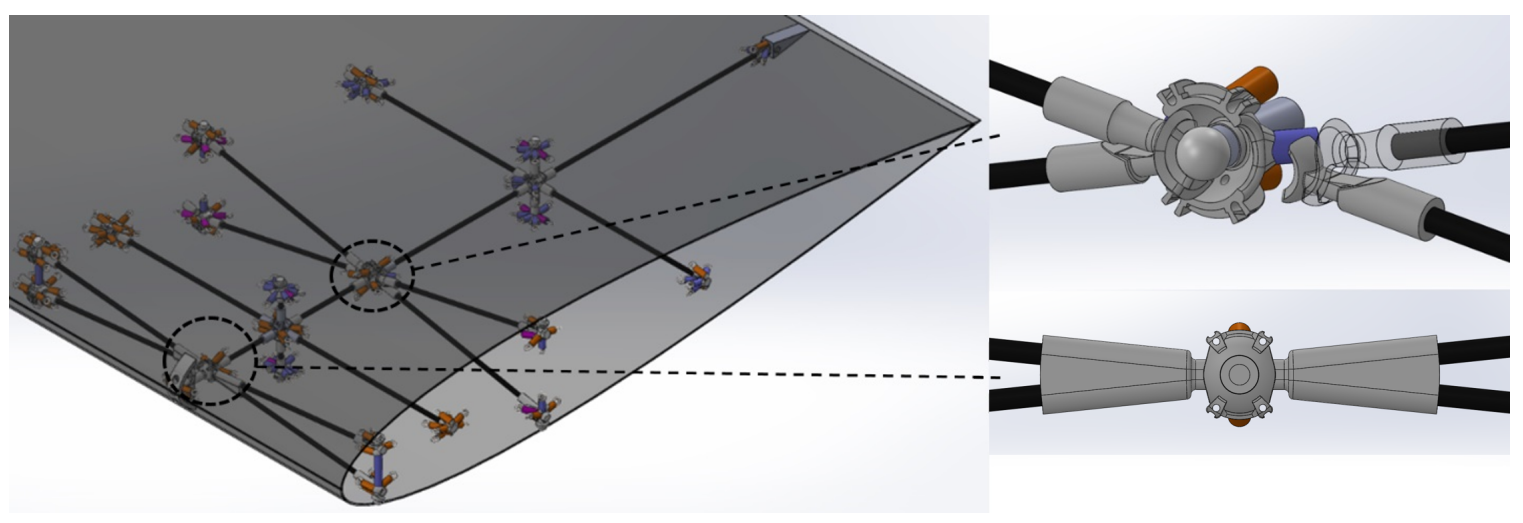

(a) Isometric view

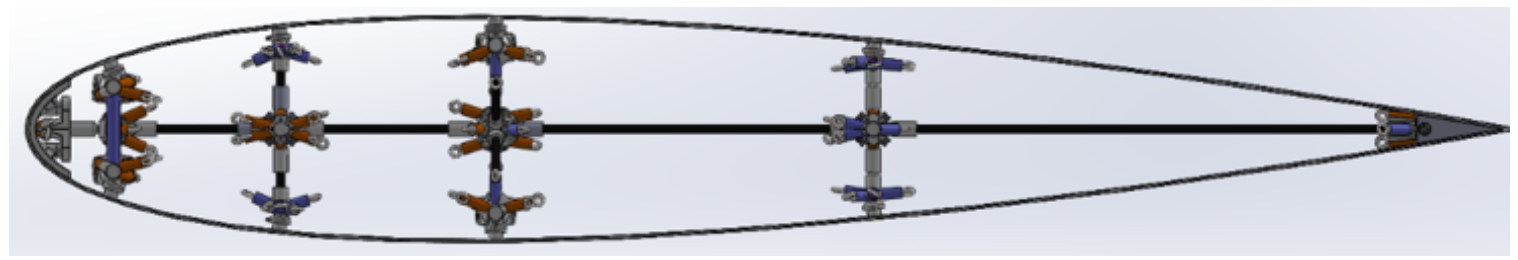

(b) Side view

Figure 6.8: Finalized tensegrity wing section design. Colors indicate the condition of the cable connections as being in-line (blue), offset (orange), or sharing a hook (purple).

interlocked while still allowing gaps for the required strut rotation about the node. This design necessitates that the two interlocking rockers be 3D printed together. The joint shown in the bottom right of Figure 6.8a experiences a narrower angle than the top joint and is compensated by combining the two rockers into one solid piece. Another design compromise is the conjoining of joints that are spaced closely together, making a cable connection infeasible.

Located at the wingtip, there are specialized connectors that close off the wing. The leading and trailing edge joints have a detachable piece that helps support the skin. Joints 
that directly touch the skin of the wing include bosses adjacent to the skin that allow a \#0 thread-forming screw to be screwed in through the skin and into the boss for a secure connection. The skin is anticipated to be of carbon fiber construction. In order to aid in attaching the skin via screws, a lip for a small ring shaped magnet is included on the previously mentioned bosses. Small magnets would be placed on the boss lips underneath the wing skin, the skin would be slid over the tensegrity frame, and then additional magnets would be used overtop the skin to aid in identifying the required locations for the screws to be inserted into the bosses. Initially one section of the wing was constructed. For future expansion the remaining three wing sections would be connected by repeated use of class 2 joints, still based on class 6 joint design, simply with only two struts instead of six.

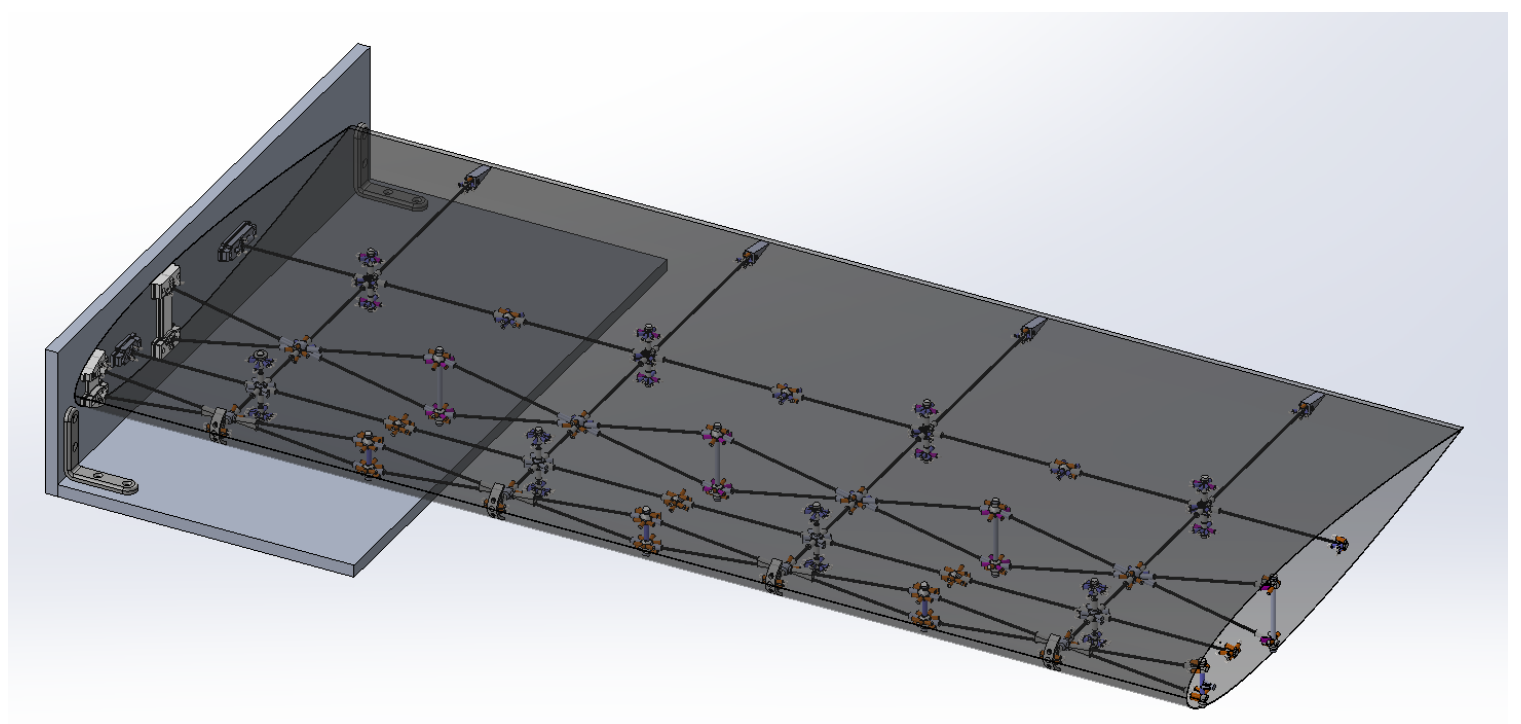

Figure 6.9: Finalized full tensegrity wing design

After the first wing section design was completed, the full tensegrity wing composed of four sections was to be fabricated with potential design changes based on feedback from the 
first section. This feedback, detailed in Section 6.4, was used to modify the existing wing design. Figure 6.9 shows the revised design of the full tensegrity wing. It features more design compromises such as fused joints that don't permit strut rotation and additional conjoining of joints that are closely located. These added design compromises were to aid in the assembly and stable pretensioning of the tensegrity wing. Due to timing and unavailability of 3D printing resources, the full wing was not fabricated.

\subsection{Final Tensegrity Wing Fabrication}

Figure 6.10a shows the fabricated tensegrity wing section completed. The previously constructed 3D model of the design was used to aid in obtaining measurements for creation of the struts and cables. The struts used were $3 \mathrm{~mm}$ (0.118 in) OD $2 \mathrm{~mm}$ (0.079 in) ID carbon fiber rods. The struts were created to proper length by roughing cutting them to a slight excess length with a Dremel, followed by repeatedly sanding them down with sandpaper and measuring them with calipers. Based on the caliper's accuracy the struts were sanded down to within $\pm 0.025 \mathrm{~mm}( \pm 0.001 \mathrm{in})$ of their required length.

Figure $6.10 \mathrm{~b}$ and Figure $6.10 \mathrm{c}$ show the two prominent cable types used. The cable used was $18-8$ stainless steel $0.79 \mathrm{~mm}(1 / 32 \mathrm{in})$ braided $7 \times 7$ wire rope. The common cables without turnbuckles have cable ends consisting of a \#2 fishing snap held in place by a 0.79 mm (1/32 in) zinc plated copper ferrule. The fishing snap is able to be opened and put on the screw eye then closed afterwards to ensure that the cable can't disconnect or fall

off from the screw eye. Certain common cables have a miniature brass turnbuckle used in tightening a cable circuit for pretensioning. The turnbuckles used have a minimum closed distance of $19.05 \mathrm{~mm}$ (0.75 in) and a maximum open distance of $28.58 \mathrm{~mm}$ (1.125 in) and 


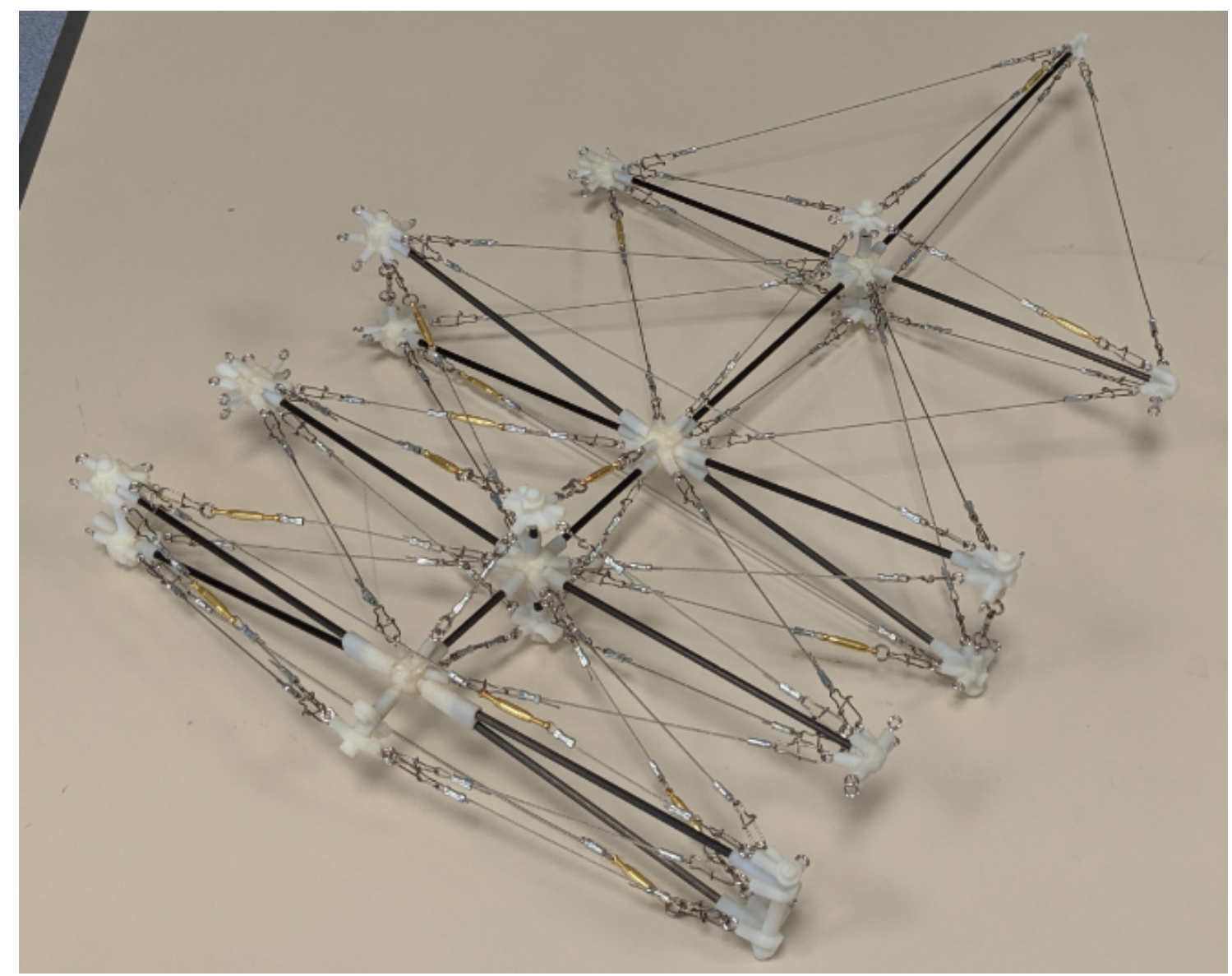

(a) Overall wing section

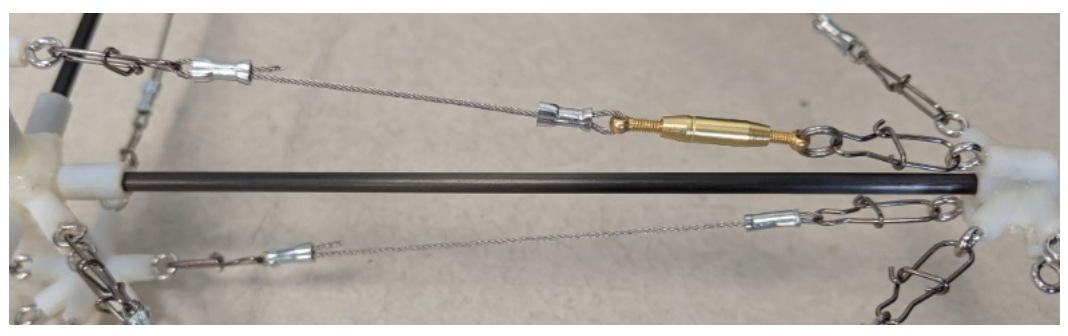

(b) Common cables

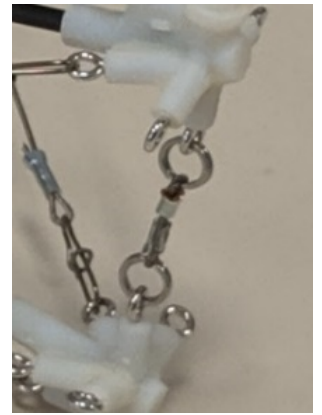

(c) Short cables

Figure 6.10: Fabricated tensegrity wing section 
are primarily used for model ships. These turnbuckles were tested and able to withstand tensile forces beyond the $253.5 \mathrm{~N}$ (57 lb) limit of the force gauge used, enough for the expected cable loads. Figure 6.10c shows the short cable used in the two locations where the joint to joint distance of $25.12 \mathrm{~mm}(0.989 ")$ was too short to permit use of the fishing snaps. To compensate for the lack of space, two $4 \mathrm{~mm}(0.157)$ split rings were used on the cable ends and the ferrules were cut in half using a Dremel to make additional space.

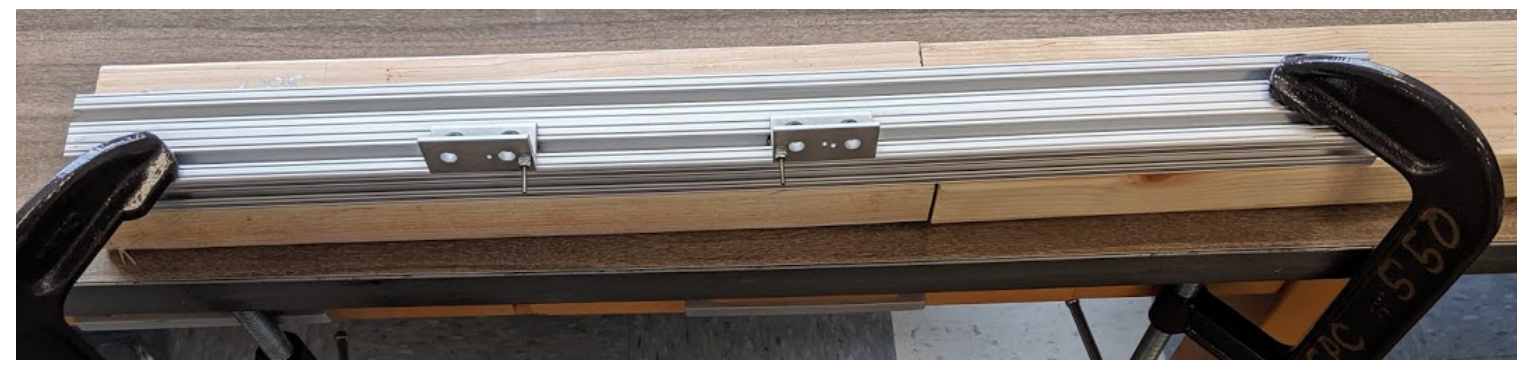

Figure 6.11: Cable fabrication fixture

Figure 6.11 shows the fixture used to aid in creating the cable lengths. Using a segment of 80/20 T-slot extruded aluminum rail, two brackets are fastened onto the rail. A \#2 machine screw is placed through a hole in each bracket and secured used four nuts. The four nuts allow a resting place for the fishing snap cable ends such that they are not right up against the bracket wall, allowing for easier use of the crimping tool for the ferrules. During cable creation one bracket is tightened down and fixed in place, then the second bracket is slid to meet the appropriate cable length as measured by the calipers, followed by it being tightened down.

As presented in Section 7.2, there were concerns with the manufacturing tolerances of the cable creation device. Addressing these concerns, a new cable creation device shown in 
Figure 6.12 was designed for future use should the full tensegrity wing ever be fabricated. The updated device consists of a Velmex Inc. $35.56 \mathrm{~cm}$ (14 in) travel manual linear stage with a $1.27 \mathrm{~mm}(0.050 \mathrm{in})$ travel per revolution. Mounted on both the stage platform and end block are L-brackets which each contain a latch tie-down hook mounted near the top of the L-bracket. The latch tie-down hooks serve as a hook to the cable fishing snaps to be attached to during the cable creation process. In use, one end of the cable that was crimped with the fishing snap would be attached to a latch tie-down hook, followed by the second fishing snap and ferrule being attached to the remaining hook while remaining uncrimped. The graduated knob of the linear stage would be adjusted to the correct cable length and then the cable's ferrule would be crimped, finishing the cable. Due to the high accuracy and precision of the linear stage this design is anticipated to perform to significantly higher accuracy than the previous design shown in Figure 6.11.

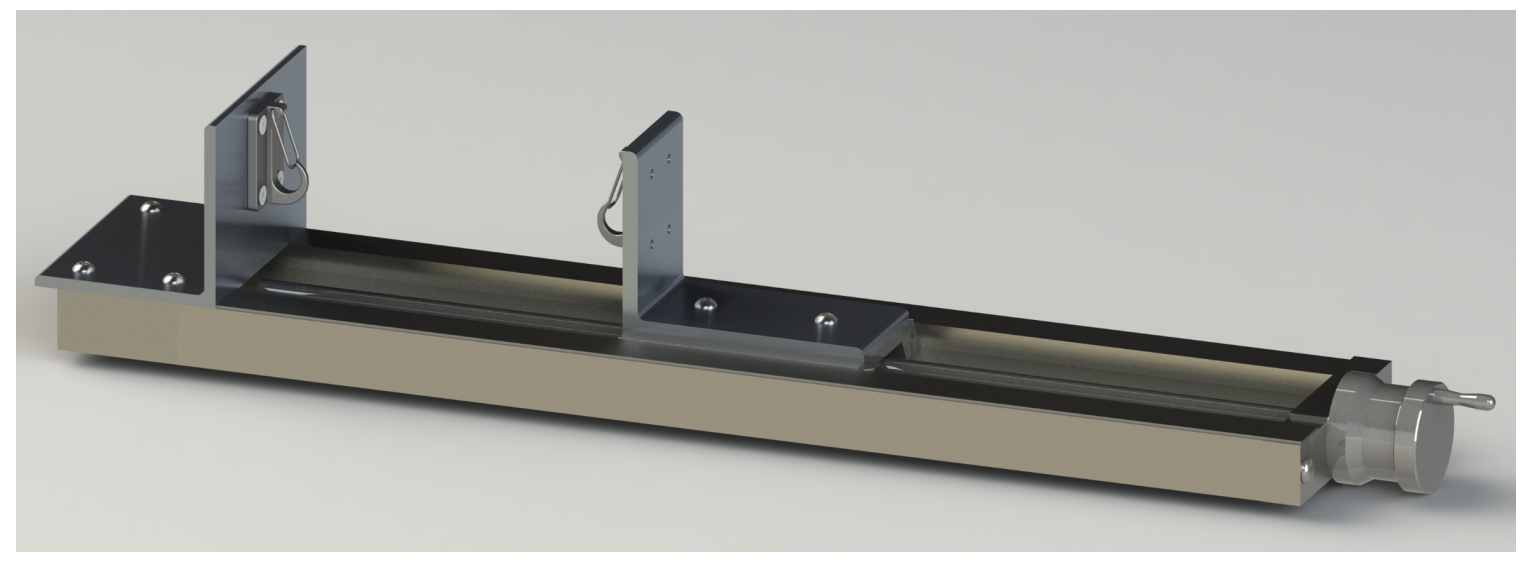

Figure 6.12: Updated cable fabrication fixture 


\section{CHAPTER VII}

\section{RESULTS AND DISCUSSION}

\subsection{Analytical Wing Comparison}

A finite element analysis-based comparison was conducted of the baseline conventional wing (RV-4), the designer-developed tensegrity wing, and topology-optimized tensegrity wing. The aerodynamic loads for the $2 \mathrm{~g}$ pullup and $1 \mathrm{~g}$ pushover maneuvers were applied to the internal structure (i.e., ribs for the RV-4 model, cable/strut nodes for the tensegrity wings) according to the interpolation scheme described in Sect. 4.3. Analyses were performed both with and without the skin, with the load application identical in each case and the skin structure simply augmenting the stiffness of the system when included.

Table 7.1: Structural comparison between the conventional wing, designer-developed tensegrity wing, and topology optimization wing.

\begin{tabular}{|c|c|c|c|c|}
\hline & \multirow[b]{2}{*}{ Conventional } & \multicolumn{2}{|c|}{ Tensegrity } \\
\hline & & & Designer-developed & Topology Optimization \\
\hline \multirow{5}{*}{ 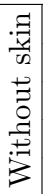 } & Mass & $10.8 \mathrm{~kg}(23.9 \mathrm{lb})$ & $6.4 \mathrm{~kg}(14.2 \mathrm{lb})$ & $5.3 \mathrm{~kg}(11.6 \mathrm{lb})$ \\
\hline & $+2 \mathrm{~g}$ max deflection & $170.9 \mathrm{~mm}(6.73 \mathrm{in})$ & $169.4 \mathrm{~mm}(6.67 \mathrm{in})$ & $170.2 \mathrm{~mm}(6.70 \mathrm{in})$ \\
\hline & $+2 \mathrm{~g}$ strain energy & $149.0 \mathrm{~N}-\mathrm{m}$ (1319 in-lb) & $172.6 \mathrm{~N}-\mathrm{m}$ (1529 in-lb) & $192.3 \mathrm{~N}-\mathrm{m}$ (1702 in-lb) \\
\hline & -1g max deflection & $-85.6 \mathrm{~mm}(-3.37 \mathrm{in})$ & $-86.9 \mathrm{~mm}(-3.42 \mathrm{in})$ & $-78.7 \mathrm{~mm}(-3.10 \mathrm{in})$ \\
\hline & -1g strain energy (no skin) & $37.3 \mathrm{~N}-\mathrm{m}$ (330 in-lb) & 43.3 N-m (383 in-lb) & 46.8 N-m (414 in-lb) \\
\hline \multirow{7}{*}{ 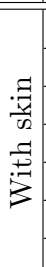 } & Mass & $25.7 \mathrm{~kg}(56.6 \mathrm{lb})$ & $21.3 \mathrm{~kg}(47.0 \mathrm{lb})$ & $20.1 \mathrm{~kg}(44.4 \mathrm{lb})$ \\
\hline & $+2 \mathrm{~g}$ max deflection & $18.3 \mathrm{~mm}(0.72 \mathrm{in})$ & $18.3 \mathrm{~mm}(0.72 \mathrm{in})$ & $16.8 \mathrm{~mm}(0.66 \mathrm{in})$ \\
\hline & $+2 \mathrm{~g}$ strain energy & 20.7 N-m (184 in-lb) & $21.9 \mathrm{~N}-\mathrm{m}$ (194.4 in-lb) & $21.5 \mathrm{~N}-\mathrm{m}$ (190 in-lb) \\
\hline & $+2 \mathrm{~g}$ max strut stress & $121 \mathrm{MPa}(17.6 \mathrm{ksi})$ & $229 \mathrm{MPa}(33.3 \mathrm{ksi})$ & $152 \mathrm{MPa}(22.0 \mathrm{ksi})$ \\
\hline & $+2 \mathrm{~g}$ max cable stress & - & $478 \mathrm{MPa}(69.4 \mathrm{ksi})$ & $218 \mathrm{MPa}(31.6 \mathrm{ksi})$ \\
\hline & -1g max deflection & $-9.1 \mathrm{~mm}(-0.36 \mathrm{in})$ & $-9.2 \mathrm{~mm}(-0.36 \mathrm{in})$ & $-8.3 \mathrm{~mm}(-0.33 \mathrm{in})$ \\
\hline & $-1 \mathrm{~g}$ strain energy & $5.2 \mathrm{~N}-\mathrm{m}(46 \mathrm{in}-\mathrm{lb})$ & $5.5 \mathrm{~N}-\mathrm{m}(48 \mathrm{in}-\mathrm{lb})$ & $5.5 \mathrm{~N}-\mathrm{m}(48$ in-lb) \\
\hline
\end{tabular}

The results of the wing comparison are presented in Table 7.1. As can be observed, the designer-developed and topology-optimized tensegrity wings are able to approximately 
match the maximum deflection of the conventional wing for each load case, with and without skin, at a lower mass. Without skin, the mass is $6.4 \mathrm{~kg}(14.2 \mathrm{lb})$ and $5.3 \mathrm{~kg}(11.6 \mathrm{lb})$ for the tensegrity wings vs. $10.8 \mathrm{~kg}(23.9 \mathrm{lb})$ for the conventional. Including the skin, the designerdeveloped and topology-optimized tensegrity wings have masses of $21.3 \mathrm{~kg}(47.0 \mathrm{lb})$ and 20.1 $\mathrm{kg}(44.4 \mathrm{lb})$ compared to $25.7 \mathrm{~kg}(56.6 \mathrm{lb})$ for the conventional wing. The strain energies of the tensegrity wing designs, however, exceed that of the conventional design. Figure 7.2 shows the vertical displacement of the conventional wing model for the $+2 \mathrm{~g}$ load condition, which served as the displacement limit for the sizing optimizations in Sections 5.3 and 5.4. In addition to modest bending, significant twisting of the conventional wing structure can be observed, with the pressure loads at the leading edge causing much higher displacements of the nose ribs than of the flap ribs. Both tensegrity wing structures exhibited bending with slight twisting, with the entire wingtip section deflecting nearly the same amount. This effect is shown in Figure 7.1 in contrast to the twisting in Figure 7.2. Since the tensegrity

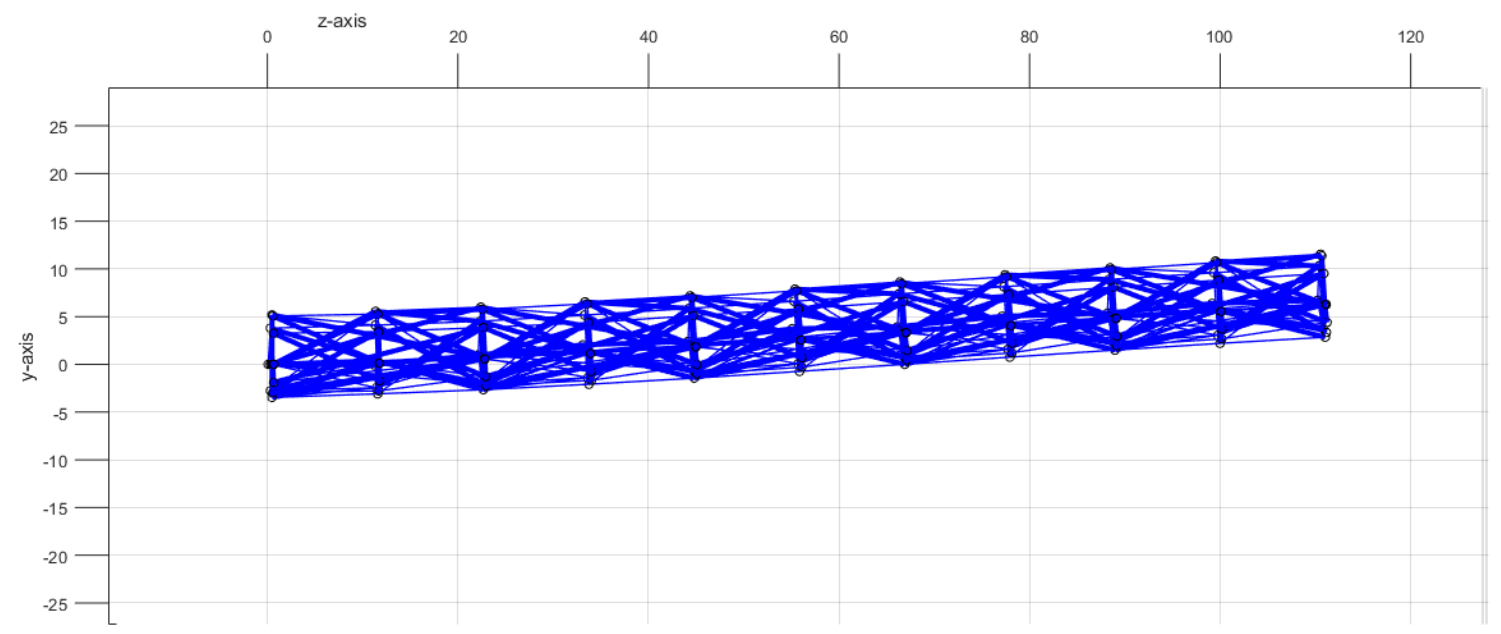

Figure 7.1: The displacements of the topology-optimized tensegrity wing under the $+2 \mathrm{~g}$ load condition 
structures were designed to approximate the maximum deflection of the conventional wing, they exhibit higher strain energy. Also note that all wings displayed essentially symmetric deflections under positive and negative load (and scaled for the $-1 \mathrm{~g}$ load condition). This linear response was not generally anticipated for tensegrity-based designs, due to the use of tension-only cables and compression-only struts, but was observed for the resulting wing structures in this study.

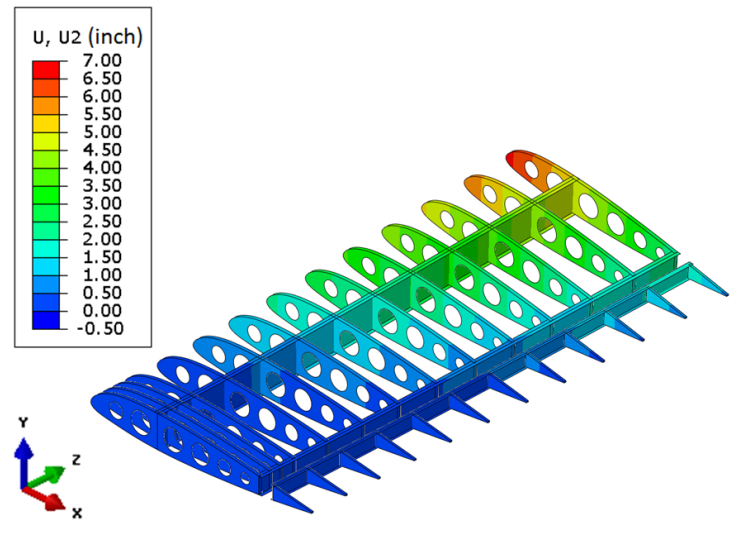

(a)

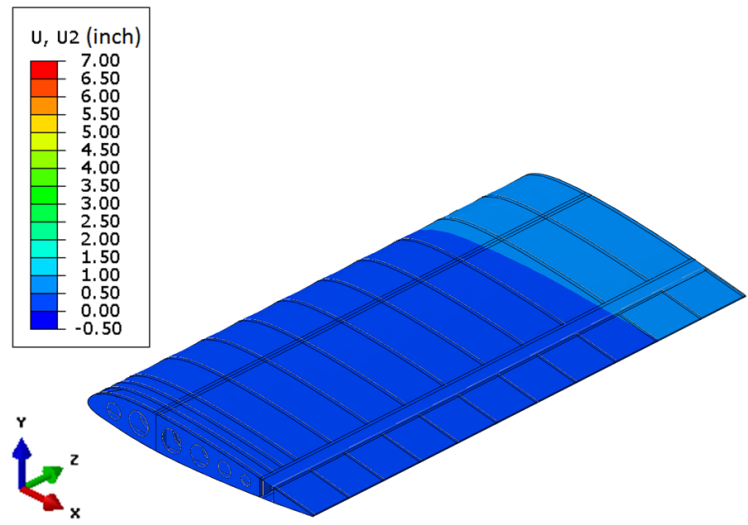

(b)

Figure 7.2: The vertical displacements of the conventional wing structure subjected to the $+2 \mathrm{~g}$ load condition, for the models (a) with no skin and (b) with the skin.

\subsection{Observations from the Tensegrity Wing Prototype Fabrication}

The design and individual components of the tensegrity wing section prototype performed as expected. In assembling the prototype, achieving and maintaining proper pretension of the cables proved difficult. Manufacturing tolerances of cable lengths is a major concern. Consistent cable pretensions are necessary to stabilize the tensegrity system into 
an equilibrium geometry. With the existing cable fabrication device cable lengths were constructed at approximately $\pm 0.25 \mathrm{~mm}( \pm 0.010 \mathrm{in})$, which proved to be insufficient accuracy. As a result of this an updated cable fabrication device shown in Figure 6.12 was designed for future use in the event the full tensegrity wing was fabricated.

In addition to the cable inaccuracies, the original concept of using a single turnbuckle in each cable loop did not appear to achieve an acceptable pretension and geometric equilibrium. At the desired pretension levels, the required cable deflections between unstressed and pretensioned states for many cables is approximately $0.025 \mathrm{~mm}(0.001 \mathrm{in})$. This small required deflection is why in cables without a turnbuckle the aforementioned current cable inaccuracies are considered significant and are presumably required to be improved to achieve equilibrium. Since the range of adjustment of the turnbuckles is $9.53 \mathrm{~mm}(0.375$ in), appropriate turnbuckle adjustments should be able to overcome the effect of cable inaccuracies, at least in the cables with a turnbuckle.

Through manual tuning of the turnbuckles, even with excess turnbuckles per cable loop, achieving the proper equilibrium for even a small section of the tensegrity was challenging and not accomplished. An inelegant solution for this would be incorporating a turnbuckle into more cables or every cable at the cost of a higher weight structure. However, the reliance on manual visual-based tuning of the turnbuckles could be inherently too challenging to achieve equilibrium with and adding additional turnbuckles could further exacerbate this effect.

Pretension methods that reduce the reliance of the visual-based tuning of the turnbuckles could produce improved results. The required cable pretension values for each cable can be determined based on measurements taken of the 3D assembly model. Therefore the use 
of a cable tension meter for measuring pretensions could provide guidance for turnbuckle adjustment, reducing the possible error from the visual-based tuning.

Unmeasured sources of deflection in the cables that counteract pretension efforts could exist such as joint clearances, slack in certain cable assemblies, and screw eye boss deformation under loading. Additionally, the pretension was observed to reduce over time. This is believed to have occurred primarily from the turnbuckles, as they were noticed to be visibly loosened over time. The turnbuckle threading is speculated to have slipped from cyclic loading or vibrations during the transportation and adjustment of the structure. A crude test for creep of the turnbuckles was conducted with a $4.5 \mathrm{~kg}(10 \mathrm{lb})$ static weight hanging vertically from a turnbuckle for 24 hours. Afterward there was no noticeable deflection of the turnbuckle. This does not entirely rule out creep as a factor in the loosening of the turnbuckles do to the small sample size and uncontrolled nature of the test.

The original plan was to conduct experimentation on the rigidity of the physical prototype. With the issues encountered in cable tensions, that experimentation was not performed. 


\section{CHAPTER VIII}

\section{CONCLUSIONS}

This thesis presents tensegrity aircraft wing concepts and compares their simulated structural performance to a conventional wing. One tensegrity wing is developed by using engineering design insights, and a second is the result of a topology optimization. The Van's RV-4 was selected to serve as the baseline conventional aircraft wing structure. The structural response of the baseline conventional structure was analytically determined for two aerodynamic loading conditions, namely a $2 \mathrm{~g}$ pullup and a $1 \mathrm{~g}$ pushover maneuver. The designer-developed tensegrity wing was configured, and the individual cables and struts

were sized, to approximately match the structural response of the Van's RV-4. A second tensegrity wing was created from a topology optimization of a one-fifth spanwise section using the ground structure method. The topology optimization results were replicated to form a full wing and a member sizing optimization was performed, as with the designerdeveloped wing, to nearly match the structural response of the Van's RV-4. Comparing the underlying structure, the resulting designer-developed tensegrity wing weighs $56 \%$ that of the baseline wing, and the topology-optimized wing weighs $49 \%$ of the baseline. Valuable design insight was gained from fabricating a tensegrity wing prototype which would be useful in realizing tensegrity structures beyond research efforts. 


\section{BIBLIOGRAPHY}

[1] N. B. H. Ali and I. Smith, "Dynamic behavior and vibration control of a tensegrity structure," International Journal of Solids and Structures, vol. 47, no. 9, pp. 1285-1296, 2010 .

[2] H. Assimi, A. Jamali, and N. Nariman-Zadeh, "Sizing and topology optimization of truss structures using genetic programming," Swarm and evolutionary computation, vol. 37, pp. 90-103, 2017.

[3] J.-L. Batoz, K.-J. Bathe, and L.-W. Ho, "A study of three-node triangular plate bending elements," International Journal for Numerical Methods in Engineering, vol. 15, no. 12 , pp. $1771-1812,1980$.

[4] J. Bruce, K. Caluwaerts, A. Iscen, A. P. Sabelhaus, and V. SunSpiral, "Design and evolution of a modular tensegrity robot platform," in 2014 IEEE International Conference on Robotics and Automation (ICRA). IEEE, 2014, pp. 3483-3489.

[5] O. S. Center, "Ohio supercomputer center," 1987. [Online]. Available: http: //osc.edu/ark:/19495/f5s1ph73

[6] P. R. Daniel et al., "Aircraft design: a conceptual approach," Published by American Institute of Aeronautics and Astronautics Inc, vol. 6, 2018.

[7] S. Ehara and Y. Kanno, "Topology design of tensegrity structures via mixed integer programming," International Journal of Solids and Structures, vol. 47, no. 5, pp. 571$579,2010$.

[8] Extra-200 Information Manual, Extra Aircraft Production and Sales, Hunxe, Germany, 1996.

[9] A. for Students, "International standard atmosphere (isa)," http :// www . aerodynamics4students.com/properties-of-the-atmosphere, 2018, accessed: 2019-0204.

[10] R. B. Fuller, "Tensile-integrity structures," U.S. Patent 3063 521A, Nov. 13, 1962.

[11] S. Guest, "The stiffness of prestressed frameworks: a unifying approach," International Journal of Solids and Structures, vol. 43, no. 3-4, pp. 842-854, 2006.

[12] E. Heartney and K. Snelson, "Kenneth snelson: Art and ideas," Available at http: //kennethsnelson.net/KennethSnelson_Art_And_Ideas.pdf (2020/03/08).

[13] A. A. S. M. Inc., "Aluminum 2024-t4," http:// asm . matweb . com / search / SpecificMaterial.asp?bassnum=MA2024T4, 2018, accessed: 2018-12-07. 
[14] V. A. Inc., "Total performance," https://www.vansaircraft.com/, 2019, accessed: 201905-15.

[15] Y. Kanno, "Topology optimization of tensegrity structures under compliance constraint: a mixed integer linear programming approach," Optimization and Engineering, vol. 14, no. 1, pp. 61-96, 2013.

[16] H. Kino, Y. Matsutani, S. Katakabe, and H. Ochi, "Prototype of a tensegrity robot with nine wires for switching locomotion and calculation method of the balancing internal force," Procedia Computer Science, vol. 105, pp. 1-6, 2017.

[17] S. Lessard, D. Castro, W. Asper, S. D. Chopra, L. B. Baltaxe-Admony, M. Teodorescu, V. SunSpiral, and A. Agogino, "A bio-inspired tensegrity manipulator with multidof, structurally compliant joints," in 2016 IEEE/RSJ International Conference on Intelligent Robots and Systems (IROS). IEEE, 2016, pp. 5515-5520.

[18] D. L. Logan, A first course in the finite element method, 5th ed. Cengage Learning, 2012.

[19] D. H. Myszka and J. J. Joo, "A study on the structural suitability of tensegrity structures to serve as morphing aircraft wings," in ASME 2018 International Design Engineering Technical Conferences and Computers and Information in Engineering Conference. American Society of Mechanical Engineers, 2018, pp. V05BT07A005V05BT07A005.

[20] D. H. Myszka, J. J. Joo, D. C. Woods, and A. P. Murray, "Topology optimization of cable-actuated, shape-changing, tensegrity systems for path generation," in ASME 2019 International Design Engineering Technical Conferences and Computers and Information in Engineering Conference. American Society of Mechanical Engineers Digital Collection.

[21] C. Niu, Airframe structural design: practical design information and data on aircraft structures. Conmilit Press Limited Hong Kong, 1999.

[22] M. I. of Technology, "Xfoil subsonic airfoil development system," http://web.mit.edu/ drela/Public/web/xfoil/, 2018, accessed: 2018-02-06.

[23] S. Pellegrino, "Analysis of prestressed mechanisms," International Journal of Solids and Structures, vol. 26, no. 12, pp. 1329-1350, 1990.

[24] S. Pellegrino and C. R. Calladine, "Matrix analysis of statically and kinematically indeterminate frameworks," International Journal of Solids and Structures, vol. 22, no. 4, pp. 409-428, 1986.

[25] R. L. Reid, "The art of science." Civil Engineering (08857024), vol. 80, no. 3, p. 68, 2010. [Online]. Available: http://libproxy.udayton.edu/login?url=http://search. ebscohost.com/login.aspx?direct $=$ true $\& \mathrm{db}=\mathrm{f} 5 \mathrm{~h} \& \mathrm{AN}=48589137 \&$ site $=$ eds-live 
[26] R. E. Skelton, "Dynamics and control of aerospace systems," 2002.

[27] R. E. Skelton and M. C. de Oliveira, Tensegrity systems. Springer, 2009, vol. 1.

[28] K. D. Snelson, "Continuous tension, discontinuous compression structures," U.S. Patent 3169611 A, Feb. 16, 1965.

[29] B. K. Stanford and P. D. Dunning, "Optimal topology of aircraft rib and spar structures under aeroelastic loads," Journal of Aircraft, vol. 52, no. 4, pp. 1298-1311, 2014.

[30] A. Tibert and S. Pellegrino, "Review of form-finding methods for tensegrity structures," International Journal of Space Structures, vol. 18, no. 4, pp. 209-223, 2003.

[31] RV-4 Construction Manual, Van's Aircraft, Inc., Aurora, Oregon, 2005.

[32] D. Walker and D. Liu, "Topology optimization of an aircraft wing," in 56th AIAA/ASCE/AHS/ASC Structures, Structural Dynamics, and Materials Conference, 2015, p. 0976.

[33] D. L. Walker, "Topology optimization of an aircraft wing," Master's thesis, Air Force Institute of Technology, Wright-Patterson AFB, OH, 72015.

[34] D. Williamson, R. E. Skelton, and J. Han, "Equilibrium conditions of a tensegrity structure," International Journal of Solids and structures, vol. 40, no. 23, pp. 6347$6367,2003$.

[35] L. A. Wolsey, Integer programming. Wiley, 1998.

[36] X. Xu, Y. Wang, and Y. Luo, "General approach for topology-finding of tensegrity structures," Journal of Structural Engineering, vol. 142, no. 10, p. 04016061, 2016.

[37] — - "An improved multi-objective topology optimization approach for tensegrity structures," Advances in Structural Engineering, vol. 21, no. 1, pp. 59-70, 2018.

[38] T. Zegard and G. H. Paulino, "Grand-ground structure based topology optimization for arbitrary $2 \mathrm{~d}$ domains using matlab," Structural and Multidisciplinary Optimization, vol. 50, no. 5, pp. 861-882, 2014.

[39] J. Zhang and M. Ohsaki, Tensegrity Structures. Springer, 2015.

[40] — - "Stability conditions for tensegrity structures," International journal of solids and structures, vol. 44, no. 11-12, pp. 3875-3886, 2007. 